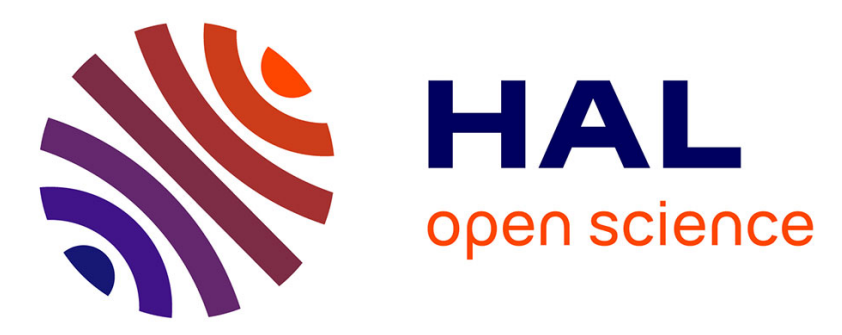

\title{
Improved bounds on the energy-minimizing strains in martensitic polycrystals
}

Michaël Peigney

\section{To cite this version:}

Michaël Peigney. Improved bounds on the energy-minimizing strains in martensitic polycrystals. Continuum Mechanics and Thermodynamics, 2016, 28 (4), pp.923-946. 10.1007/s00161-015-0427-4 . hal-01254058

\section{HAL Id: hal-01254058 \\ https://hal-enpc.archives-ouvertes.fr/hal-01254058}

Submitted on 11 Jan 2016

HAL is a multi-disciplinary open access archive for the deposit and dissemination of scientific research documents, whether they are published or not. The documents may come from teaching and research institutions in France or abroad, or from public or private research centers.
L'archive ouverte pluridisciplinaire $\mathbf{H A L}$, est destinée au dépôt et à la diffusion de documents scientifiques de niveau recherche, publiés ou non, émanant des établissements d'enseignement et de recherche français ou étrangers, des laboratoires publics ou privés. 


\title{
Michaël Peigney \\ Improved bounds on the energy-minimizing strains in martensitic polycrystals
}

\begin{abstract}
This paper is concerned with the theoretical prediction of the energy-minimizing (or recoverable) strains in martensitic polycrystals, considering a nonlinear elasticity model of phase transformation at finite strains. The main results are some rigorous upper bounds on the set of energy-minimizing strains. Those bounds depend on the polycrystalline texture through the volume fractions of the different orientations. The simplest form of the bounds presented is obtained by combining recent results for single crystals with a homogenization approach proposed previously for martensitic polycrystals. However, the polycrystalline bound delivered by that procedure may fail to recover the monocrystalline bound in the homogeneous limit, as is demonstrated in this paper by considering an example related to tetragonal martensite. This motivates the development of a more detailed analysis, leading to improved polycrystalline bounds that are notably consistent with results for single crystals in the homogeneous limit. A two-orientation polycrystal of tetragonal martensite is studied as an illustration. In that case, analytical expressions of the upper bounds are derived and the results are compared with lower bounds obtained by considering laminate textures.
\end{abstract}

Keywords Shape memory alloys $\cdot$ Polycrystal $\cdot$ Energy minimization $\cdot$ Finite strains

PACS 46.25.g $\cdot 62.20 . \mathrm{fg} \cdot 81.30 . \mathrm{fk}$

\section{Introduction}

The peculiar properties of shape memory alloys (SMAs) - such as the shape memory effect or the superelastic behavior - are the macroscopic result of a diffusionless solid/solid phase transformation between different crystallographic structures, known as austenite and martensite [20,4]. Since the austenite has a crystallographic structure with higher symmetry than the martensite, several martensitic variants need to be distinguished depending on the orientation of the martensitic lattice with respect to the austenitic lattice. Each martensitic variant is characterized by a transformation strain that is defined as the deformation gradient between the austenitic and martensitic lattices. The number of martensitic variants and the corresponding transformation strains depend on the alloy considered. $\mathrm{MnCu}$ and $\mathrm{MnNi}$ are examples of alloys undergoing a cubic to tetragonal transformation (i.e. the austenite has a cubic lattice and the martensite has a tetragonal lattice). In that case, there are 3 martensitic variants. Widely used alloys such as NiTi or $\gamma \mathrm{CuAlNi}$ obey a cubic-to-monoclinic transformation, in which case there are 12 martensitic variants to be considered.

The phase transformation between austenite and martensite can be triggered both by mechanical and thermal loading. The most striking illustration of that phenomenon is the shape memory effect displayed by alloys such as NiTi or CuAlNi: cooling down a stress-free sample below a critical temperature $T^{0}$ transforms the

Michaël Peigney

Université Paris-Est, Laboratoire Navier (UMR 8205), CNRS, Ecole des Ponts ParisTech, IFSTTAR, F-77455 Marne la vallée, France

E-mail: michael.peigney@polytechnique.org 
homogeneous austenite (stable at high temperature) to a martensitic microstructure, in which the martensitic variants arrange themselves so as to produce a stress- free state with no macroscopic deformation. Once in the cooled state, deforming the sample mechanically entails a phase transformation of some martensitic variants to others. After unloading, a macroscopic residual stress-free strain is observed as a result from the cooperative effect of the microscopic transformation strains in each variant. Heating the sample transforms the martensite back in austenite, thus restoring the initial configuration. We note that, in a polycrystalline sample (which is the common form of commercially available SMAs), the formation of austenite/martensite microstructures is typically observed in each grain. This leads one to distinguish between three length scales: the microscopic scale of the austenite/martensite microstructures, the mesoscopic scale of the constitutive grains, and the macroscopic scale of the polycrystal consisting in numerous grains. The macroscopic scale can be interpreted as the length scale of a representative volume element (RVE) of the polycrystal. Expressing the macroscopic free energy in terms of the mesoscopic free energies in the constitutive grains is a long standing topic in the study of polycrystals and granular materials (see e.g. [18] for a recent work in the field of granular materials).

A possible route to study the formation of microstructures in SMAs is to adopt a nonlinear elasticity model of phase transformation [2]. The general principle is that, under a prescribed loading, the system tends to minimize its free energy. This approach amounts to consider that the evolutions are thermodynamically reversible and to look for stable equilibria. Assuming the microscopic, mesoscopic and macroscopic scales to be well separated, the energy minimization principle leads to different expressions of the free energy at each scale. Denoting the microscopic free energy by $\Psi$, the mesoscopic energy $\tilde{\Psi}$ is obtained as the relaxation (or quasiconvexification) of $\Psi$, which essentially amounts to solve an optimal design problem with respect to the martensite/austenite geometric arrangement (see Section 2 for a precise definition). Viewing group of grains with the same orientation as individual homogeneous materials (governed by mesoscopic free energies), the polycrystal can be regarded as a composite material with a macroscopic energy $\bar{\Psi}$ obtained by homogenization of the constitutive free energy functions.

Assuming the microscopic free energy $\Psi$ to be known, determining its relaxation $\tilde{\Psi}$ largely remains an open problem. Estimating the macroscopic free energy $\bar{\Psi}$ is even more challenging as stress and strain compatibility conditions between the grains need to be taken into account. Of special interest are the strains that minimize the mesoscopic (resp. macroscopic) free-energy. Those energy-minimizing strains can indeed be interpreted as the recoverable strains of a monocrystalline (resp. polycrystalline) shape memory alloy, i.e. the strains that can be recovered by the shape memory effect [6]. Knowing the set of recoverable strains is crucial for designing SMA systems. Experiments only give partial insight in the structure of that set, as they usually only give measurements along prescribed directions (see e.g. [33]). In this paper, we propose theoretical bounds on the whole set of recoverable strains, i.e. in the space of three-dimensional deformation gradients. Those bounds are expressed in terms of the lattice parameters and of statistical information on the polycrystalline texture (namely the orientation distribution function). Such data can be obtained experimentally using X-ray diffraction or EBSD (Electron Back Scattering Diffraction).

The problem can be formulated either in the geometrically nonlinear setting or in the geometrically linear setting. The latter assumes small deformations with respect to a reference configuration. Although that assumption can be regarded as questionable for shape memory alloys (notably because of possibly large rotation effect), it allows the analysis to be simplified significantly. In the geometrically linear setting, the exact expression of the relaxation of a (piecewise quadratic) double-well energy can been obtained [15,27]. Lower and upper bounds have been proposed for the relaxation of a three- or more-well energy [29,11,10,23]. The structure of the set of mesoscopic energy-minimizing strains has been studied in detail, notably for the most challenging case of monoclinic martensite $[6,7,25]$. Concerning polycrystals, bounds are available for the macroscopic energy-minimizing strains as well as for the macroscopic free energy [12,23,6,28].

In the geometrically non linear setting, the set of mesoscopic energy-minimizing strains has been obtained in closed-form for a double-well energy [2]. Using known restrictions on Young measure, an upper bound on the mesoscopic energy-minimizing strains has been proposed in the case of three or more wells [24]. Regarding polycrystals, a general method has been introduced in [22] for generating upper bounds on the set of macroscopic energy-minimizing strains, assuming that the set of energy-minimizing strains of the constitutive single crystals (or at least an upper bound on it) is known. The approach used in [22] is based on the translation method [16], which has proved to be a powerful tool in various problems related to homogenization. In particular, for nonlinear composites, variations of that method allows one to obtain bounds on the macroscopic strains that are compatible with a given macroscopic stress [17,30,21]. 
In this paper, we focus on upper bounds of the macroscopic energy-minimizing strains of martensitic polycrystals, in the geometrically non-linear setting. We begin in Section 2 by describing the nonlinear elasticity model of phase transformation and presenting the upper bound of [24] for single crystals. In Section 3 we combine the monocrystalline bound with the methodology of [22] to derive explicit upper bounds for polycrystals. It turns out, however, that the upper bounds that are obtained may fail to recover the single crystal bound in the homogeneous limit, as discussed in Section 4. Motivated by that observation, we modify the polycrystalline approach so as to take the special structure of the single crystal bound into account (Section 5). This results in new upper bounds for polycrystals, which improve on the bounds of Section 3 and are consistent with the single crystal bound in the homogeneous limit. In Section 6, we study a two-orientation/three-well polycrystal and compare the proposed upper bounds with lamination lower bounds.

\section{Single crystal}

In the framework of nonlinear elasticity at finite strains, the microscopic behavior of a shape-memory alloy is described by its free energy density $\Psi$, which is a function of the deformation gradient $\mathbf{F}$. We denote by $\mathcal{K}$ the set of deformation gradients that minimize $\Psi$. Without loss of generality, we can assume that the minimum value of $\Psi$ is equal to 0 , so that $\Psi \geq 0$ and

$$
\mathcal{K}=\{\mathbf{F} \mid \Psi(\mathbf{F})=0\} .
$$

The principle of frame indifference implies that $\Psi(\mathbf{R} . \mathbf{F})=\Psi(\mathbf{F})$ for any rotation $\mathbf{R}$ and deformation gradient $\mathbf{F}$. Moreover, in the case of shape memory alloys, $\Psi$ has a multi well structure. Accordingly, the set $\mathcal{K}$ takes the form

$$
\mathcal{K}=\bigcup_{i=1}^{m} \operatorname{SO}(3) \mathbf{U}_{i}
$$

where $\mathbf{U}_{1}, \cdots, \mathbf{U}_{m}$ are given symmetric positive definite tensors that depend both on the temperature and on the alloy considered. At a temperature $T$ below the transformation temperature $T^{0}$, the number $m$ of wells is equal to the number $n v$ of martensitic variants. The corresponding transformation strains $\mathbf{U}_{1}, \cdots, \mathbf{U}_{m}$ are all symmetry related, i.e. for any $(i, j)$ there exists a rotation $\mathbf{R}_{i j}$ such that $\mathbf{U}_{j}={ }^{\mathrm{t}} \mathbf{R}_{i j} \cdot \mathbf{U}_{i} \cdot \mathbf{R}_{i j}$ (here and in the following, the pre-superscript ${ }^{\mathrm{t}}$ denotes the transpose operator). At $T=T^{0}$, both the austenite and the martensite variants minimize the microscopic energy, so that $m=n v+1$. In general, the deformation gradient $\mathbf{U}_{n v+1}$ corresponding to the austenite is not symmetry related to any of the martensitic transformation strain. At $T>T^{0}$, there is only one well which corresponds to the austenite phase.

Consider a reference configuration where a domain $\Omega$ is occupied by a single crystal of shape memory alloy. Each material particle is identified by its location $\mathbf{x}$ in the reference configuration. The deformation of the crystal is described by a mapping $\mathbf{u}: \Omega \mapsto \mathbb{R}^{3}$ which gives the location (in the deformed configuration) of the material particle $\mathbf{x}$. We study the equilibrium of the crystal under boundary conditions of the form $\mathbf{u}(\mathbf{x})=\tilde{\mathbf{F}} . \mathbf{x}$ where $\tilde{\mathbf{F}}$ is a given mesoscopic deformation gradient (here and in the following, the superscript . denotes quantities defined at the mesoscopic scale). The effective free energy at equilibrium is given by

$$
\tilde{\Psi}(\tilde{\mathbf{F}})=\inf _{\mathbf{F} \in \mathscr{A}(\tilde{\mathbf{F}})}\langle\Psi(\mathbf{F})\rangle
$$

where $\langle$.$\rangle denotes volume average over the domain \Omega$ and the set $\mathscr{A}(\tilde{\mathbf{F}})$ of admissible deformation gradient fields is defined by

$$
\mathscr{A}(\tilde{\mathbf{F}})=\left\{\mathbf{F} \mid \operatorname{det} \mathbf{F}>0, \exists \mathbf{u}(\mathbf{x}) \in W^{1, \infty}\left(\Omega, \mathbb{R}^{3}\right) \text { such that } \mathbf{F}=\nabla \mathbf{u} \text { in } \Omega ; \mathbf{u}(\mathbf{x})=\tilde{\mathbf{F}} . \mathbf{x} \text { on } \partial \Omega\right\} .
$$

The infimum problem in (1) is easily solved if $\tilde{\mathbf{F}} \in \mathcal{K}:$ in that case, the homogeneous field $\mathbf{F}(\mathbf{x})=\tilde{\mathbf{F}}$ realizes the infimum and we have $\tilde{\Psi}(\tilde{\mathbf{F}})=\Psi(\tilde{\mathbf{F}})=0$. The situation gets more complicated if $\tilde{\mathbf{F}} \notin \mathcal{K}$ : in that case, a minimizer of (1) does not necessarily exist. This is a consequence of the multi well structure of $\Psi$.The non existence of a minimizer physically corresponds to the formation of microstructures at a very fine scale (twinned laminates are examples of microstructures which are frequently observed in shape memory alloys, see e.g. [20]). In such a situation, the evaluation of $\tilde{\Psi}(\tilde{\mathbf{F}})$ requires to study the weak convergence of minimizing sequences, which involves mathematical concepts such as Young measures [14] and quasiconvexity [8]. The function $\tilde{\mathbf{F}} \mapsto \tilde{\Psi}(\tilde{\mathbf{F}})$ is mathematically referred to as the quasiconvexification (or relaxation) of $\Psi$ and can be 
interpreted as the mesoscopic energy of the material. Let $\tilde{\mathcal{K}}$ be the set of deformation gradients that minimize $\tilde{\Psi}$. Since $\tilde{\Psi}$ is positive and vanishes on $\mathcal{K}$, the minimum value of $\tilde{\Psi}$ is equal to 0 and we have

$$
\tilde{\mathcal{K}}=\{\tilde{\mathbf{F}} \mid \tilde{\Psi}(\tilde{\mathbf{F}})=0\} .
$$

The set $\tilde{\mathcal{K}}$ is also known as the quasiconvex hull of $\mathcal{K}[8,19]$. The exact calculation of $\tilde{\mathcal{K}}$ still remains a largely open question. In the framework of finite strains as considered in this paper, the problem has been solved exactly $[2,5]$ only in the case where

(i) the strains $\mathbf{U}_{1}, \cdots, \mathbf{U}_{m}$ have same determinant,

(ii) there exists $\mu>0$ and $\mathbf{v} \in \mathbb{R}^{3}$ such that $\mathbf{U}_{i} \mathbf{v}=\mu \mathbf{v}$ for all $1 \leq i \leq m$.

The assumption (3ii) show that problems of the form (3) are fundamentally two-dimensional. Commonly used shape-memory alloys, such as TiNi or CuAlNi, do not fulfill the assumption (3ii) and the exact expression of $\mathcal{K}$ for such materials is unknown. In such a situation, one may look for bounds (in the sense of inclusion of sets) to obtain some rigorous information on $\tilde{\mathcal{K}}$. In this paper we primarily focus on upper bounds (the question of constructing lower bounds is addressed in Section 6).

Upper bounds on $\tilde{\mathcal{K}}$ can be obtained using known restrictions on Young measures [24]. Some notations are in order. We introduce the frame-indifferent function $\Phi: \mathbb{R}^{3 \times 3} \mapsto \mathbb{R}$ defined by

$$
\Phi(\mathbf{M})=\max _{\mathbf{R} \in \operatorname{SO}(3)} \operatorname{tr}(\mathbf{R} \cdot \mathbf{M}) .
$$

The supremum problem defining $\Phi(\mathbf{M})$ can be solved in closed form : we have indeed

$$
\Phi(\mathbf{M})=\lambda_{3}+\lambda_{2}+\lambda_{1} \operatorname{sgn}(\operatorname{det} \mathbf{M})
$$

where $0 \leq \lambda_{1} \leq \lambda_{2} \leq \lambda_{3}$ are the eigenvalues of $\sqrt{{ }^{t} \mathbf{M} . \mathbf{M}}$.

Let $\mathbf{F}^{*}$ denote the adjugate of a matrix $\mathbf{F} \in \mathbb{R}^{3 \times 3}$, i.e. $\mathbf{F}^{*}=\operatorname{det} \mathbf{F}$. ${ }^{t} \mathbf{F}^{-1}$. For any $\mathbf{a} \in \mathbb{R}^{3 \times 3}, \mathbf{b} \in \mathbb{R}^{3 \times 3}$ and $c \in \mathbb{R}$, the function $h$ defined by

$$
h(\mathbf{F})=\operatorname{tr}\left(\mathbf{F} \cdot \mathbf{a}+\mathbf{F}^{*} \cdot \mathbf{b}\right)+c \operatorname{det} \mathbf{F}
$$

is known to be quasiconvex [8], i.e. satisfies

$$
h(\tilde{\mathbf{F}}) \leq\langle h(\mathbf{F})\rangle \text { for all } \tilde{\mathbf{F}} \text { and } \mathbf{F} \in \mathscr{A}(\tilde{\mathbf{F}}) .
$$

The functions $\Phi$ in (4) and $h$ in (6) are related by the following identity, which will be frequently used in this paper:

$$
\sup _{\mathbf{R} \in \mathrm{SO}(3)} h(\mathbf{R} \cdot \mathbf{F})=\Phi\left(\mathbf{F} \cdot \mathbf{a}+\mathbf{F}^{*} \cdot \mathbf{b}\right)+c \operatorname{det} \mathbf{F} .
$$

Finally, we set

$$
\mathcal{T}=\left\{\theta=\left(\theta_{1}, \cdots, \theta_{m}\right) \in \mathbb{R}^{m}: \theta_{i} \geq 0 ; \sum_{i=1}^{m} \theta_{i}=1\right\} .
$$

Using those notations, it can be proved [24] that the set $\tilde{\mathcal{K}}_{+}$defined by

$$
\begin{aligned}
& \tilde{\mathcal{K}}_{+}=\left\{\tilde{\mathbf{F}} \in \mathbb{R}^{3 \times 3}: \exists \theta \in \mathcal{T}\right. \text { such that } \operatorname{det} \tilde{\mathbf{F}}=\sum_{i=1}^{m} \theta_{i} \operatorname{det} \mathbf{U}_{i} \\
&\text { and } \left.0 \geq \sup _{(\mathbf{a}, \mathbf{b}) \in \mathscr{C}}\left\{\Phi\left(\tilde{\mathbf{F}} \cdot \mathbf{a}+\tilde{\mathbf{F}}^{*} \cdot \mathbf{b}\right)-\sum_{i=1}^{m} \theta_{i} \Phi\left(\mathbf{U}_{i} \cdot \mathbf{a}+\mathbf{U}_{i}^{*} \cdot \mathbf{b}\right)\right\}\right\}
\end{aligned}
$$

is an upper bound on $\tilde{\mathcal{K}}$, i.e. satisfies $\tilde{\mathcal{K}} \subset \tilde{\mathcal{K}}_{+}$. For a given $\tilde{\mathbf{F}}$ in $\tilde{\mathcal{K}}_{+}$, the vector $\theta$ in $(10)$ can be interpreted as the volume fractions of the different wells in a microstructure realizing $\tilde{\mathbf{F}}$. In general there is no uniqueness for $\theta$. This is connected to the fact that, except in special cases, energy-minimizing deformation gradients $\tilde{\mathbf{F}}$ can be realized by several microstructures.

In (10), $\mathscr{C}$ is a given arbitrary subset of $\mathbb{R}^{3 \times 3} \times \mathbb{R}^{3 \times 3}$. Note that each choice of $\mathscr{C}$ generates a corresponding bound on $\tilde{\mathcal{K}}$. In principle, the best bound is obtained by taking $\mathscr{C}$ as large as possible, i.e. $\mathscr{C}=\mathbb{R}^{3 \times 3} \times \mathbb{R}^{3 \times 3}$. 
In practice, however, one may restrict $\mathscr{C}$ to a smaller family to ease the calculations and notably obtain closed form expressions.

For later reference, we close this section with a brief derivation of the bound $\tilde{\mathcal{K}}_{+}$. A fundamental property of $\tilde{\mathcal{K}}$ is that any given $\tilde{\mathbf{F}} \in \tilde{\mathcal{K}}$ can be written as

$$
\tilde{\mathbf{F}}=\int_{\mathbb{R}^{3 \times 3}} \mathbf{A} d v(\mathbf{A})
$$

for some Young measure $v$ supported on $\mathcal{K}[2,19]$. For our purpose, we only need to record the following properties of Young measures [14]:

$$
\begin{aligned}
v & \geq 0, \int_{\mathbb{R}^{3 \times 3}} d v(\mathbf{A})=1 \\
\text { (ii) } f\left(\int_{\mathbb{R}^{3 \times 3}} \mathbf{A} d v(\mathbf{A})\right) & \leq \int_{\mathbb{R}^{3 \times 3}} f(\mathbf{A}) d v(\mathbf{A}) \text { for any } f \text { quasiconvex. }
\end{aligned}
$$

Moreover, since $\mathcal{K}=\cup_{i=1}^{m} \mathcal{K}_{i}$ where the wells $\mathcal{K}_{i}=\operatorname{SO}(3) \mathbf{U}_{i}$ are closed and disjoint, the Young measure $v$ in (11) can be written as

$$
v=\sum_{i=1}^{m} v_{i}
$$

where $v_{i}$ is a positive measure supported on $\mathcal{K}_{i}$ [2]. Using the properties (11-12) with quasiconvex functions $h$ of the form (6), we obtain

$$
h(\tilde{\mathbf{F}}) \leq \sum_{i=1}^{m} \int_{\mathrm{SO}(3) \mathbf{U}_{i}} h(\mathbf{A}) d v_{i}(\mathbf{A}) .
$$

The identity (8) gives $h(\mathbf{A}) \leq \Phi\left(\mathbf{U}_{i} \cdot \mathbf{a}+\mathbf{U}_{i}^{*} \cdot \mathbf{b}\right)+c \operatorname{det} \mathbf{U}_{i}$ for any $\mathbf{A} \in \operatorname{SO}(3) \mathbf{U}_{i}$. Consequently we find

$$
h(\tilde{\mathbf{F}}) \leq \sum_{i=1}^{m} \theta_{i}\left\{\Phi\left(\mathbf{U}_{i} \cdot \mathbf{a}+\mathbf{U}_{i}^{*} \cdot \mathbf{b}\right)+c \operatorname{det} \mathbf{U}_{i}\right\}
$$

where $\theta_{i}=\int_{\mathbb{R}^{3 \times 3}} d v_{i}(\mathbf{A})$. As a consequence of $(12 i)$, the vector $\theta=\left(\theta_{1}, \cdots, \theta_{n}\right)$ belongs to the set $\mathcal{T}$ in (9).

Let now $\tilde{\mathbf{R}}$ be an arbitrary rotation. Observing that $\mathbf{F} \mapsto h(\tilde{\mathbf{R}} . \mathbf{F})$ is quasiconvex and proceeding as in (13), we obtain

$$
h(\tilde{\mathbf{R}} . \tilde{\mathbf{F}}) \leq \sum_{i=1}^{m} \theta_{i}\left\{\Phi\left(\mathbf{U}_{i} \cdot \mathbf{a}+\mathbf{U}_{i}^{*} \cdot \mathbf{b}\right)+c \operatorname{det} \mathbf{U}_{i}\right\} .
$$

Taking the supremum over $\tilde{\mathbf{R}}$ in this last equation and using the identity (8), we get

$$
\Phi\left(\tilde{\mathbf{F}} \cdot \mathbf{a}+\tilde{\mathbf{F}}^{*} \cdot \mathbf{b}\right)+c \operatorname{det} \tilde{\mathbf{F}} \leq \sum_{i=1}^{m} \theta_{i}\left\{\Phi\left(\mathbf{U}_{i} \cdot \mathbf{a}+\mathbf{U}_{i}^{*} \cdot \mathbf{b}\right)+c \operatorname{det} \mathbf{U}_{i}\right\}
$$

The conclusion is that for any $\tilde{\mathbf{F}} \in \tilde{\mathcal{K}}$, there exists $\theta \in \mathcal{T}$ such that (14) holds for any $(\mathbf{a}, \mathbf{b}, c)$. In other words, the set $\tilde{\mathcal{K}}_{+}$in $(10)$ is an upper bound on $\tilde{\mathcal{K}}$.

As detailed in [24], the bound given by (10) coincides with $\tilde{\mathcal{K}}$ for the reference cases where the exact expression of $\tilde{\mathcal{K}}$ is available, such as problems of the form (3). 


\section{Polycrystal}

Now consider a polycrystal occupying a domain $\Omega$. We can decompose $\Omega$ as $\Omega=\cup_{r=1}^{n} \Omega^{r}$ where each subdomain $\Omega^{r}$ is formed by grains with the same orientation. The microscopic free energy in $\Omega^{r}$ can be written as

$$
\Psi^{r}(\mathbf{F})=\Psi\left({ }^{t} \mathbf{R}^{r} . \mathbf{F} \cdot \mathbf{R}^{r}\right)
$$

where $\mathbf{R}^{r}$ is a rotation describing the orientation in $\Omega^{r}$ relative to a reference single crystal. Defining $\chi^{r}$ the characteristic function of $\Omega^{r}$ (i.e. $\chi^{r}(\mathbf{x})=1$ if $\mathbf{x} \in \Omega^{r}$, and $\chi^{r}(\mathbf{x})=0$ otherwise), the microscopic free energy of the polycrystal is a heterogeneous function of the form

$$
\Psi(\mathbf{F}, \mathbf{x})=\sum_{r=1}^{n} \chi^{r}(\mathbf{x}) \Psi^{r}(\mathbf{F})
$$

The set $\mathcal{K}(\mathbf{x})$ of deformation gradients minimizing $\mathbf{F} \mapsto \Psi(\mathbf{F}, \mathbf{x})$ is thus

$$
\mathcal{K}(\mathbf{x})=\bigcup_{i=1}^{m} \operatorname{SO}(3) \mathbf{U}_{i}(\mathbf{x})
$$

where $\mathbf{U}_{i}(\mathbf{x})=\sum_{r=1}^{n} \chi^{r}(\mathbf{x}) \mathbf{U}_{i}^{r}$ and $\mathbf{U}_{i}^{r}=\mathbf{R}^{r} . \mathbf{U}_{i} .{ }^{\mathrm{t}} \mathbf{R}^{r}$. The functions $\chi^{1}, \cdots, \chi^{n}$ together with the rotations $\mathbf{R}^{1}, \cdots, \mathbf{R}^{n}$ fully describe the texture of the polycrystal, i.e. the shapes, distributions and orientations of the grains.

Consider boundary conditions of the form $\mathbf{u}(\mathbf{x})=\overline{\mathbf{F}} \cdot \mathbf{x}$ where $\overline{\mathbf{F}}$ is a given macroscopic deformation gradient (here and in the following, the superscript ${ }^{-}$denotes macroscopic quantities). At equilibrium, one expects the formation of microstructures in each grain. Assuming the scale of such microstructures to be much smaller than the scale of the grains, the macroscopic free energy $\bar{\Psi}(\overline{\mathbf{F}})$ of the polycrystal is given by

$$
\bar{\Psi}(\overline{\mathbf{F}})=\min _{\mathbf{F} \in \mathscr{A}(\overline{\mathbf{F}})}\left\langle\sum_{r=1}^{n} \chi^{r} \tilde{\Psi}^{r}(\mathbf{F})\right\rangle
$$

where $\tilde{\Psi}^{r}$ is the relaxation of $\Psi^{r}$, as defined in (1) (see e.g.[4] for a detailed justification). In the following, we primarily focus on the set $\overline{\mathcal{K}}$ of deformation gradients that minimize the macroscopic energy, i.e.

$$
\overline{\mathcal{K}}=\{\overline{\mathbf{F}} \mid \bar{\Psi}(\overline{\mathbf{F}})=0\} .
$$

In view of (17), we have the following characterization of $\overline{\mathcal{K}}$ :

$$
\overline{\mathcal{K}}=\{\overline{\mathbf{F}} \mid \exists \mathbf{F} \in \mathscr{A}(\overline{\mathbf{F}}) ; \mathbf{F}(\mathbf{x}) \in \tilde{\mathcal{K}}(\mathbf{x}) \text { for all } \mathbf{x} \in \Omega\} .
$$

Hence the distinctive property of strains $\overline{\mathbf{F}}$ in $\overline{\mathcal{K}}$ is that they can be realized by a deformation $\mathbf{u}(\mathbf{x})$ whose gradient $\mathbf{F}=\nabla \mathbf{u}$ satisfies the local constraint $\mathbf{F}(\mathbf{x}) \in \tilde{\mathcal{K}}(\mathbf{x})$ at each point.

Let $\tilde{\mathcal{K}}^{r}$ be the quasi convex hull of $\cup_{i=1}^{m} \mathrm{SO}(3) \mathbf{U}_{i}^{r}$, i.e. the set of strains minimizing $\tilde{\Psi}^{r}$. For any $\overline{\mathbf{F}}$ in $\cap_{r=1}^{n} \tilde{\mathcal{K}}^{r}$, the homogeneous field $\mathbf{F}(\mathbf{x})=\overline{\mathbf{F}}$ is in $\mathscr{A}(\overline{\mathbf{F}})$ and satisfies the constraint $\mathbf{F}(\mathbf{x}) \in \tilde{\mathcal{K}}(\mathbf{x})$ in $\Omega$. The set $\overline{\mathcal{K}}_{-}=\cap_{r=1}^{n} \tilde{\mathcal{K}}^{r}$ is thus a lower bound on $\overline{\mathcal{K}}$, in the sense that $\overline{\mathcal{K}}-\subset \overline{\mathcal{K}}$. That lower bound (referred to as the Taylor bound) has been studied in detail in the geometrically linear setting [6]. Note that the bound $\overline{\mathcal{K}}$ only depends on the rotations $\mathbf{R}^{1}, \cdots, \mathbf{R}^{n}$, and not on the functions $\chi^{1}, \cdots, \chi^{n}$, therefore ignoring a lot of information about the texture.

Using the quasiconvexity of the function $h$ in (6), upper bounds on $\overline{\mathcal{K}}$ that take one-point statistics of the functions $\chi^{r}$ can be derived [22]. Let indeed $\overline{\mathbf{F}}$ be in $\overline{\mathcal{K}}$ and consider a deformation gradient field $\mathbf{F}$ in $\mathscr{A}(\overline{\mathbf{F}})$ such that $\mathbf{F}(\mathbf{x}) \in \tilde{\mathcal{K}}(\mathbf{x})$ for all $\mathbf{x}$ (recall that such $\mathbf{F}$ exists by (18)). Using the property (7) and noting that $\sum_{r} \chi^{r}=1$, we have

$$
h(\overline{\mathbf{F}}) \leq\langle h(\mathbf{F})\rangle=\sum_{r=1}^{n}\left\langle\chi^{r} h(\mathbf{F})\right\rangle .
$$

Observe from (8) that $h(\mathbf{F}(\mathbf{x})) \leq \Phi\left(\mathbf{F}(\mathbf{x}) \cdot \mathbf{a}+\mathbf{F}^{*}(\mathbf{x}) \cdot \mathbf{b}\right)+c \operatorname{det} \mathbf{F}(\mathbf{x})$. For $\mathbf{x} \in \Omega^{r}$, we have $\mathbf{F}(\mathbf{x}) \in \tilde{\mathcal{K}}^{r}$ and consequently

$$
h(\mathbf{F}(\mathbf{x})) \leq \Phi\left(\mathbf{F}(\mathbf{x}) \cdot \mathbf{a}+\mathbf{F}^{*}(\mathbf{x}) \cdot \mathbf{b}\right)+c \operatorname{det} \mathbf{F}(\mathbf{x}) \leq \sup _{\mathbf{F} \in \tilde{\mathcal{K}}^{r}}\left\{\Phi\left(\mathbf{F} \cdot \mathbf{a}+\mathbf{F}^{*} \cdot \mathbf{b}\right)+c \operatorname{det} \mathbf{F}\right\} .
$$


Substituting in (19) gives

$$
h(\overline{\mathbf{F}}) \leq \sum_{r=1}^{n}\left\{\left\langle\chi^{r}\right\rangle \sup _{\mathbf{F} \in \tilde{\mathcal{K}}^{r}}\left\{\Phi\left(\mathbf{F} \cdot \mathbf{a}+\mathbf{F}^{*} \cdot \mathbf{b}\right)+c \operatorname{det} \mathbf{F}\right\}\right\} .
$$

The frame-indifference of $\overline{\mathcal{K}}$ implies that $\mathbf{R} . \overline{\mathbf{F}}$ also satisfies (20) for any rotation $\mathbf{R}$, which using (8) leads to

$$
\Phi\left(\overline{\mathbf{F}} . \mathbf{a}+\overline{\mathbf{F}}^{*} . \mathbf{b}\right)+c \operatorname{det} \overline{\mathbf{F}} \leq \sum_{r=1}^{n}\left\{\left\langle\chi^{r}\right\rangle \sup _{\mathbf{F} \in \tilde{\mathcal{K}}^{r}}\left\{\Phi\left(\mathbf{F} . \mathbf{a}+\mathbf{F}^{*} \cdot \mathbf{b}\right)+c \operatorname{det} \mathbf{F}\right\}\right\} .
$$

Let $\mathscr{C}^{\prime}$ denote a given subset of $\mathbb{R}^{3 \times 3} \times \mathbb{R}^{3 \times 3}$. From (21) we obtain that any $\overline{\mathbf{F}}$ in $\overline{\mathcal{K}}$ necessarily satisfies

$$
\sup _{c} \sup _{(\mathbf{a}, \mathbf{b}) \in \mathscr{C}^{\prime}}\left\{\Phi\left(\overline{\mathbf{F}} \cdot \mathbf{a}+\overline{\mathbf{F}}^{*} \cdot \mathbf{b}\right)+c \operatorname{det} \overline{\mathbf{F}}-\sum_{r=1}^{n}\left\langle\chi^{r}\right\rangle \sup _{\mathbf{F} \in \tilde{\mathcal{K}}^{r}}\left\{\Phi\left(\mathbf{F} \cdot \mathbf{a}+\mathbf{F}^{*} \cdot \mathbf{b}\right)+c \operatorname{det} \mathbf{F}\right\}\right\} \leq 0 .
$$

The set of deformation gradient $\overline{\mathbf{F}}$ verifying (22) is thus an upper bound of $\overline{\mathcal{K}}$. That upper bound has been used in [22] on some examples where the constitutive single crystals fulfill the conditions (3), in which case the sets $\tilde{\mathcal{K}}^{r}$ are known. For polycrystals that do not satisfy (3), the direct application of the bound (22) is hampered by the fact that $\tilde{\mathcal{K}}^{r}$ is unknown. In such case, calculating the supremum over $\tilde{\mathcal{K}}^{r}$ that appears in (22) remains out of reach. Such a difficulty can be overcome by using the results from Section 2 . Let indeed $\tilde{\mathcal{K}}_{+}^{r}$ be the upper bound of $\tilde{\mathcal{K}}^{r}$ defined in Eq. (10). Since $\tilde{\mathcal{K}}^{r} \subset \tilde{\mathcal{K}}_{+}^{r}$, we have $\sup _{\tilde{\mathcal{K}}^{r}} \Phi\left(\mathbf{F} . \mathbf{a}+\mathbf{F}^{*} . \mathbf{b}\right)+c \operatorname{det} \mathbf{F} \leq$ $\sup _{\tilde{\mathcal{K}}_{+}^{r}} \Phi\left(\mathbf{F} . \mathbf{a}+\mathbf{F}^{*} \cdot \mathbf{b}\right)+c \operatorname{det} \mathbf{F}$. Therefore, we obtain from (22) that any $\overline{\mathbf{F}}$ in $\overline{\mathcal{K}}$ necessarily satisfies

$$
\sup _{c} \sup _{(\mathbf{a}, \mathbf{b}) \in \mathscr{C}^{\prime}}\left\{\Phi\left(\overline{\mathbf{F}} \cdot \mathbf{a}+\overline{\mathbf{F}}^{*} \cdot \mathbf{b}\right)+c \operatorname{det} \overline{\mathbf{U}}-\sum_{r=1}^{n}\left\langle\chi^{r}\right\rangle \sup _{\mathbf{F} \in \tilde{\mathcal{K}}_{+}^{r}}\left\{\Phi\left(\mathbf{F} . \mathbf{a}+\mathbf{F}^{*} \cdot \mathbf{b}\right)+c \operatorname{det} \mathbf{F}\right\}\right\} \leq 0 .
$$

The calculation of the right-hand side in (23) can be further simplified if $\mathscr{C}^{\prime}=\mathscr{C}$, i.e. if the bound (23) and the bound $\tilde{\mathcal{K}}_{+}^{r}$ given by (10) and are calculated using the same set of tensors $(\mathbf{a}, \mathbf{b})$. In that case, we have indeed

$$
\sup _{\mathbf{F} \in \tilde{\mathcal{K}}_{+}^{r}}\left\{\Phi\left(\mathbf{F} . \mathbf{a}+\mathbf{F}^{*} \cdot \mathbf{b}\right)+c \operatorname{det} \mathbf{F}\right\}=\max _{1 \leq i \leq m}\left\{\Phi\left(\mathbf{U}_{i}^{r} \cdot \mathbf{a}+\mathbf{U}_{i}^{r, *} \cdot \mathbf{b}\right)+c \operatorname{det} \mathbf{U}_{i}^{r}\right\}
$$

for any $(\mathbf{a}, \mathbf{b}) \in \mathscr{C}$. That last property is easily proved: for a given $\mathbf{F}$ in $\tilde{\mathcal{K}}_{+}^{r}$, Eq. (10) yields

$$
\Phi\left(\mathbf{F} . \mathbf{a}+\mathbf{F}^{*} . \mathbf{b}\right)+c \operatorname{det} \mathbf{F} \leq \sum_{i=1}^{m} \theta_{i}\left\{\Phi\left(\mathbf{U}_{i}^{r} \cdot \mathbf{a}+\mathbf{U}_{i}^{r, *} \cdot \mathbf{b}\right)+c \operatorname{det} \mathbf{U}_{i}^{r}\right\}
$$

for some $\theta \in \mathcal{T}$. Since any $\theta$ in $\mathcal{T}$ verifies $\theta_{i} \geq 0$ and $\sum_{i} \theta_{i}=1$, the right hand side of (25) is bounded from above by $\max _{1 \leq i \leq m}\left\{\Phi\left(\mathbf{U}_{i}^{r} \cdot \mathbf{a}+\mathbf{U}_{i}^{r, *} \cdot \mathbf{b}\right)+c \operatorname{det} \overline{\mathbf{U}}_{i}^{r}\right\}$. Therefore we have

$$
\sup _{\mathbf{F} \in \tilde{\mathcal{K}}_{+}^{r}}\left\{\Phi\left(\mathbf{F} . \mathbf{a}+\mathbf{F}^{*} \cdot \mathbf{b}\right)+c \operatorname{det} \mathbf{F}\right\} \leq \max _{1 \leq i \leq m}\left\{\Phi\left(\mathbf{U}_{i}^{r} \cdot \mathbf{a}+\mathbf{U}_{i}^{r * *} \cdot \mathbf{b}\right)+c \operatorname{det} \mathbf{U}_{i}^{r}\right\}
$$

The converse inequality follows directly from the fact that $\mathbf{U}_{i}^{r} \in \tilde{\mathcal{K}}_{+}^{r}$ for $i=1, \cdots, m$.

Combining (23) and (24) gives

$$
\overline{\mathcal{K}} \subset \overline{\mathcal{K}}_{+}^{0}
$$

where

$\overline{\mathcal{K}}_{+}^{0}=\left\{\overline{\mathbf{F}} \in \mathbb{R}^{3 \times 3}: 0 \geq \sup _{c} \sup _{(\mathbf{a}, \mathbf{b}) \in \mathscr{C}}\left\{\Phi\left(\overline{\mathbf{F}} \cdot \mathbf{a}+\overline{\mathbf{F}}^{*} \cdot \mathbf{b}\right)+c \operatorname{det} \overline{\mathbf{F}}-\sum_{r=1}^{n}\left\langle\chi^{r}\right\rangle \max _{1 \leq i \leq m}\left\{\Phi\left(\mathbf{U}_{i}^{r} \cdot \mathbf{a}+\mathbf{U}_{i}^{r, *} \cdot \mathbf{b}\right)+c \operatorname{det} \mathbf{U}_{i}^{r}\right\}\right\}\right\}$. 
In the most usual situation when all the transformation strains $\mathbf{U}_{i}^{r}$ have the same determinant, the set $\overline{\mathcal{K}}_{+}^{0}$ can be rewritten more simply as

$$
\overline{\mathcal{K}}_{+}^{0}=\left\{\overline{\mathbf{F}} \in \mathbb{R}^{3 \times 3}: \operatorname{det} \overline{\mathbf{F}}=\operatorname{det} \mathbf{U}_{1}^{1}, 0 \geq \sup _{(\mathbf{a}, \mathbf{b}) \in \mathscr{C}}\left\{\Phi\left(\overline{\mathbf{F}} \cdot \mathbf{a}+\overline{\mathbf{F}}^{*} \cdot \mathbf{b}\right)-\sum_{r=1}^{n}\left\langle\chi^{r}\right\rangle \max _{1 \leq i \leq m} \Phi\left(\mathbf{U}_{i}^{r} \cdot \mathbf{a}+\mathbf{U}_{i}^{r * *} \cdot \mathbf{b}\right)\right\}\right\} .
$$

The set $\overline{\mathcal{K}}_{+}^{0}$ is an explicit upper bound that depends on one-point statistics of the texture, i.e. on the volume fractions $\left\langle\chi^{r}\right\rangle$ of the different orientations.

For a discrete $\mathscr{C}$, checking if a given deformation gradient $\overline{\mathbf{F}}$ is in $\overline{\mathcal{K}}_{+}^{0}$ amounts to check if $\overline{\mathbf{F}}$ satisfies a finite number of constraints. A similar remark holds if $\mathscr{C}$ is not discrete but with the form

$$
\bigcup_{j=1}^{N} \bigcup_{\mathbf{w} \in \mathbb{R}^{3}}\left\{\left(\mathbf{u}_{j} \otimes \mathbf{w}, \mathbf{u}_{j}^{\prime} \otimes \mathbf{w}\right)\right\}
$$

In that case, Eq. (5) indeed shows that $\Phi\left(\overline{\mathbf{F}} \cdot \mathbf{u}_{j} \otimes \mathbf{w}+\overline{\mathbf{F}}^{*} \cdot \mathbf{u}_{j}^{\prime} \otimes \mathbf{w}\right)=\left\|\overline{\mathbf{F}} \cdot \mathbf{u}_{j}+\overline{\mathbf{F}}^{*} \cdot \mathbf{u}_{j}^{\prime}\right\| \cdot\|\mathbf{w}\|$ so that the condition

$$
0 \geq \sup _{(\mathbf{a}, \mathbf{b}) \in \mathscr{C}}\left\{\Phi\left(\overline{\mathbf{F}} \cdot \mathbf{a}+\overline{\mathbf{F}}^{*} \cdot \mathbf{b}\right)-\sum_{r=1}^{n}\left\langle\chi^{r}\right\rangle \max _{1 \leq i \leq m} \Phi\left(\mathbf{U}_{i}^{r} \cdot \mathbf{a}+\mathbf{U}_{i}^{r * *} \cdot \mathbf{b}\right)\right\}
$$

is equivalent to

$$
0 \geq \max _{1 \leq j \leq N}\left\{\left\|\overline{\mathbf{F}} \cdot \mathbf{u}_{j}+\overline{\mathbf{F}}^{*} \cdot \mathbf{u}_{j}^{\prime}\right\|-\sum_{r=1}^{n}\left\langle\chi^{r}\right\rangle \max _{1 \leq i \leq m}\left\|\mathbf{U}_{i}^{r} \cdot \mathbf{u}_{j}+\mathbf{U}_{i}^{r * *} \cdot \mathbf{u}_{j}^{\prime}\right\|\right\}
$$

i.e. to a set of $N$ constraints on $\overline{\mathbf{F}}$.

\section{Homogeneous limit}

In this Section we study the behavior of the polycrystalline bound (27) in the limit $\left\langle\chi^{1}\right\rangle \rightarrow 1$. In that case, the set $\overline{\mathcal{K}}_{+}^{0}$ in Eq. (27) is characterized by

$$
0 \geq \sup _{c} \sup _{(\mathbf{a}, \mathbf{b}) \in \mathscr{C}}\left\{\Phi\left(\overline{\mathbf{F}} \cdot \mathbf{a}+\overline{\mathbf{F}}^{*} \cdot \mathbf{b}\right)+c \operatorname{det} \overline{\mathbf{F}}-\max _{1 \leq i \leq m}\left\{\Phi\left(\mathbf{U}_{i}^{1} \cdot \mathbf{a}+\mathbf{U}_{i}^{1, *} \cdot \mathbf{b}\right)+c \operatorname{det} \mathbf{U}_{i}^{1}\right\}\right\} .
$$

In the homogeneous limit $\left\langle\chi^{1}\right\rangle \rightarrow 1$, there is no distinction between the mesoscopic scale and the macroscopic scale. Since the bound $\overline{\mathcal{K}}_{+}^{0}$ is the result of substituting the monocrystalline upper bound (10) in the general prescription (22), one would expect the set defined by (29) to coincide with the monocrystalline bound $\tilde{\mathcal{K}}_{+}$ generated from the same family $\mathscr{C}$. This can be proved to be indeed the case for problems of the form (3), but it is not true in general. To illustrate that point, consider the cubic to tetragonal transformation at $T<T^{0}$ : we have $\mathcal{K}^{1}=\bigcup_{i=1}^{3} \mathrm{SO}(3) \mathbf{U}_{i}^{1}$ where

$$
\mathbf{U}_{1}^{1}=\left(\begin{array}{ccc}
\eta_{2} & 0 & 0 \\
0 & \eta_{1} & 0 \\
0 & 0 & \eta_{1}
\end{array}\right), \mathbf{U}_{2}^{1}=\left(\begin{array}{ccc}
\eta_{1} & 0 & 0 \\
0 & \eta_{2} & 0 \\
0 & 0 & \eta_{1}
\end{array}\right), \mathbf{U}_{3}^{1}=\left(\begin{array}{ccc}
\eta_{1} & 0 & 0 \\
0 & \eta_{1} & 0 \\
0 & 0 & \eta_{2}
\end{array}\right)
$$

These matrix representations are relative to the reference orthonormal basis $\left(\mathbf{v}_{1}, \mathbf{v}_{2}, \mathbf{v}_{3}\right)$ of the cubic austenitic lattice in orientation 1 . The parameters $\left(\eta_{1}, \eta_{2}\right)$ are non-negative and distinct. We assume in the following that $\eta_{2}<\eta_{1}$.

We determine the bounds $\tilde{\mathcal{K}}_{+}$in $(10)$ and $\overline{\mathcal{K}}_{+}^{0}$ in (29) corresponding to the family $\mathscr{C}$ of tensors $(\mathbf{a}, \mathbf{b})$ defined by

$$
\mathscr{C}=\bigcup_{j \in\{1,2,3\}, \mathbf{w} \in \mathbb{R}^{3}}\left\{\left(\mathbf{v}_{j} \otimes \mathbf{w}, 0\right),\left(0, \mathbf{v}_{j} \otimes \mathbf{w}\right)\right\}
$$


Let us first determine the set $\tilde{\mathcal{K}}_{+}$in (10). For a fixed $\tilde{\mathbf{F}}$ in $\tilde{\mathcal{K}}_{+}$, we can see from (10) that $\operatorname{det} \tilde{\mathbf{F}}=\eta_{1}^{2} \eta_{2}$. Moreover, there exists $\theta \in \mathcal{T}$ such that

$$
0 \geq \Phi\left(\tilde{\mathbf{F}} \cdot \mathbf{a}+\tilde{\mathbf{F}}^{*} \cdot \mathbf{b}\right)-\sum_{i=1}^{3} \theta_{i} \Phi\left(\mathbf{U}_{i}^{1} \cdot \mathbf{a}+\mathbf{U}_{i}^{1, *} \cdot \mathbf{b}\right)
$$

for all $(\mathbf{a}, \mathbf{b})$ in the family $\mathscr{C}$ defined by (31). Any such $(\mathbf{a}, \mathbf{b})$ can either be written as $\left(\mathbf{v}_{j} \otimes \mathbf{w}, 0\right)$ or $\left(0, \mathbf{v}_{j} \otimes \mathbf{w}\right)$. In the first case, the definition (4) gives $\Phi\left(\mathbf{F} . \mathbf{a}+\mathbf{F}^{*} . \mathbf{b}\right)=\left\|\mathbf{F} . \mathbf{v}_{j}\right\| .\|\mathbf{w}\|$ so that (32) becomes

$$
0 \geq\left\|\tilde{\mathbf{F}} \cdot \mathbf{v}_{j}\right\|-\sum_{i=1}^{3} \theta_{i}\left\|\mathbf{U}_{i}^{1} \cdot \mathbf{v}_{j}\right\| .
$$

Observing that $\left\|\mathbf{U}_{i}^{1} \cdot \mathbf{v}_{j}\right\|=\eta_{1}+\left(\eta_{2}-\eta_{1}\right) \delta_{i j}$ and recalling that $\sum_{i=1}^{3} \theta_{i}=1$, we obtain

$$
0 \geq\left\|\tilde{\mathbf{F}} \cdot \mathbf{v}_{j}\right\|-\eta_{1}-\theta_{j}\left(\eta_{2}-\eta_{1}\right)
$$

In the case $(\mathbf{a}, \mathbf{b})=\left(0, \mathbf{v}_{j} \otimes \mathbf{w}\right)$, a similar reasoning leads to

$$
0 \geq\left\|\tilde{\mathbf{F}}^{*} \cdot \mathbf{v}_{j}\right\|-\eta_{1} \eta_{2}-\theta_{j} \eta_{1}\left(\eta_{1}-\eta_{2}\right)
$$

This inequalities (33) and (34) can be combined as as

$$
\frac{\left\|\tilde{\mathbf{F}}^{*} \cdot \mathbf{v}_{j}\right\|-\eta_{1} \eta_{2}}{\eta_{1}\left(\eta_{1}-\eta_{2}\right)} \leq \theta_{j} \leq \frac{\eta_{1}-\left\|\tilde{\mathbf{F}} \cdot \mathbf{v}_{j}\right\|}{\eta_{1}-\eta_{2}} .
$$

Any $\theta$ in $\mathcal{T}$ is such that $\theta_{j} \geq 0$ and $\sum_{j=1}^{3} \theta_{j}=1$. It follows that any $\tilde{\mathbf{F}}$ in $\tilde{\mathcal{K}}_{+}$satisfies

$$
\begin{gathered}
0 \leq \frac{\eta_{1}-\left\|\tilde{\mathbf{F}} \cdot \mathbf{v}_{j}\right\|}{\eta_{1}-\eta_{2}} ; \frac{\left\|\tilde{\mathbf{F}}^{*} \cdot \mathbf{v}_{j}\right\|-\eta_{1} \eta_{2}}{\eta_{1}\left(\eta_{1}-\eta_{2}\right)} \leq 1 ; \frac{\left\|\tilde{\mathbf{F}}^{*} \cdot \mathbf{v}_{j}\right\|-\eta_{1} \eta_{2}}{\eta_{1}\left(\eta_{1}-\eta_{2}\right)} \leq \frac{\eta_{1}-\left\|\tilde{\mathbf{F}} \cdot \mathbf{v}_{j}\right\|}{\eta_{1}-\eta_{2}} \text { for } j=1,2,3 ; \\
-3 \eta_{2}+\frac{1}{\eta_{1}} \sum_{j=1}^{3}\left\|\tilde{\mathbf{F}}^{*} \cdot \mathbf{v}_{i}\right\| \leq \eta_{1}-\eta_{2} \leq 3 \eta_{1}-\sum_{j=1}^{3}\left\|\tilde{\mathbf{F}} \cdot \mathbf{v}_{j}\right\|
\end{gathered}
$$

which can be rewritten more simply as

$$
\begin{gathered}
\left\|\tilde{\mathbf{F}} \cdot \mathbf{v}_{j}\right\| \leq \eta_{1},\left\|\tilde{\mathbf{F}}^{*} \cdot \mathbf{v}_{j}\right\| \leq \eta_{1}^{2}, \eta_{1}\left\|\tilde{\mathbf{F}} \cdot \mathbf{v}_{j}\right\|+\left\|\tilde{\mathbf{F}}^{*} \cdot \mathbf{v}_{j}\right\| \leq \eta_{1}\left(\eta_{1}+\eta_{2}\right) \text { for } j=1,2,3 \\
\sum_{j=1}^{3}\left\|\tilde{\mathbf{F}} \cdot \mathbf{v}_{j}\right\| \leq 2 \eta_{1}+\eta_{2}, \sum_{j=1}^{3}\left\|\tilde{\mathbf{F}}^{*} \cdot \mathbf{v}_{j}\right\| \leq \eta_{1}\left(2 \eta_{2}+\eta_{1}\right)
\end{gathered}
$$

Conversely, for any $\tilde{\mathbf{F}}$ satisfying (37), it can be verified that there exists $\theta \in \mathcal{T}$ satisfying (32) for all (a,b) in $\mathscr{C}$. Therefore, the inequalities (37) (complemented by the restriction $\operatorname{det} \tilde{\mathbf{F}}=\eta_{1}^{2} \eta_{2}$ ) characterize the upper bound $\tilde{\mathcal{K}}_{+}$that is generated by the family $\mathscr{C}$ defined in Eq. (31).

Let us now calculate the bound $\overline{\mathcal{K}}_{+}^{0}$ in (29). Since the transformation strains $\mathbf{U}_{1}^{1}, \mathbf{U}_{2}^{1}, \mathbf{U}_{3}^{1}$ in (30) have same determinant, we obtain from (29) that any $\tilde{\mathbf{F}}$ in $\overline{\mathcal{K}}_{+}^{0}$ satisfies $\operatorname{det} \tilde{\mathbf{F}}=\eta_{1}^{2} \eta_{2}$ and

$$
0 \geq \Phi\left(\tilde{\mathbf{F}} \cdot \mathbf{a}+\tilde{\mathbf{F}}^{*} \cdot \mathbf{b}\right)-\max _{1 \leq i \leq 3} \Phi\left(\mathbf{U}_{i}^{1} \cdot \mathbf{a}+\mathbf{U}_{i}^{1, *} \cdot \mathbf{b}\right)
$$

for all $(\mathbf{a}, \mathbf{b})$ in $\mathscr{C}$. Using the special form of the tensors $(\mathbf{a}, \mathbf{b})$ defined in (31) and the corresponding values of $\Phi\left(\mathbf{U}_{i}^{1} \cdot \mathbf{a}+\mathbf{U}_{i}^{1, *} \cdot \mathbf{b}\right)$, the inequality (38) is found to be equivalent to

$$
\left\|\tilde{\mathbf{F}} . \mathbf{v}_{j}\right\| \leq \eta_{1},\left\|\tilde{\mathbf{F}}^{*} \cdot \mathbf{v}_{j}\right\| \leq \eta_{1}^{2} \text { for } j=1,2,3 \text {. }
$$

Comparing (39) with (37) shows that $\tilde{\mathcal{K}}_{+} \subset \overline{\mathcal{K}}_{+}^{0}$. That inclusion is actually strict: consider indeed the deformation gradient $\tilde{\mathbf{F}}_{0}$ defined by

$$
\tilde{\mathbf{F}}_{0}=\left(\eta_{1}^{2} \eta_{2}\right)^{1 / 3}\left(\mathbf{I}+\left(\sqrt{\left(\frac{\eta_{1}}{\eta_{2}}\right)^{2 / 3}-1}\right) \mathbf{v}_{1} \otimes \mathbf{v}_{2}\right)
$$


We have $\operatorname{det} \tilde{\mathbf{F}}_{0}=\eta_{1}^{2} \eta_{2}$ and

$$
\left\|\tilde{\mathbf{F}}_{0} \cdot \mathbf{v}_{1}\right\|=\left\|\tilde{\mathbf{F}}_{0} \cdot \mathbf{v}_{3}\right\|=\left(\eta_{1}^{2} \eta_{2}\right)^{1 / 3},\left\|\tilde{\mathbf{F}}_{0} \cdot \mathbf{v}_{2}\right\|=\eta_{1},\left\|\tilde{\mathbf{F}}_{0}^{*} \cdot \mathbf{v}_{2}\right\|=\left\|\tilde{\mathbf{F}}_{0}^{*} \cdot \mathbf{v}_{3}\right\|=\left(\eta_{1}^{2} \eta_{2}\right)^{2 / 3},\left\|\tilde{\mathbf{F}}_{0}^{*} \cdot \mathbf{v}_{1}\right\|=\eta_{1}^{5 / 3} \eta_{2}^{1 / 3} .
$$

Since $\eta_{2}<\eta_{1}$, it can easily be verified that $\tilde{\mathbf{F}}_{0}$ satisfies (39) and therefore is in $\overline{\mathcal{K}}_{+}^{0}$. However, we have $\eta_{1}\left\|\tilde{\mathbf{F}}_{0} \cdot \mathbf{v}_{2}\right\|+\left\|\tilde{\mathbf{F}}_{0}^{*} \cdot \mathbf{v}_{2}\right\|=\eta_{1}^{2}+\left(\eta_{1}^{2} \eta_{2}\right)^{2 / 3}>\eta_{1}\left(\eta_{1}+\eta_{2}\right)$. Therefore, $\tilde{\mathbf{F}}_{0}$ does not satisfy (37) and is not in $\tilde{\mathcal{K}}_{+}$.

This example shows that the bound in (27) may fail to recover the single crystal bound in the homogeneous limit. This is an indication that some information is lost when directly plugging the monocrystalline bound (10) in the general prescription (22). In the following, we derive an improved upper bound on $\overline{\mathcal{K}}$ that notably coincides with the single crystal bound (10) in the homogeneous limit.

\section{Improved bound for polycrystals}

The bound $\overline{\mathcal{K}}_{+}^{0}$ in (27) can be improved upon by taking the special structure of the monocrystalline bound (10) into account, as is now explained. Consider a given $\overline{\mathbf{F}}$ in $\overline{\mathcal{K}}$. By (18), there exists a field $\mathbf{F} \in \mathscr{A}(\overline{\mathbf{F}})$ such that $\mathbf{F}(\mathbf{x}) \in \tilde{\mathcal{K}}(\mathbf{x})$ for all $\mathbf{x} \in \Omega$. Recall that $\mathcal{K}(\mathbf{x})=\bigcup_{i=1}^{m} \operatorname{SO}(3) \mathbf{U}_{i}(\mathbf{x})$ where $\mathbf{U}_{i}(\mathbf{x})=\sum_{r=1}^{n} \chi^{r}(\mathbf{x}) \mathbf{U}_{i}^{r}$. Using the bound (10) on $\tilde{\mathcal{K}}(\mathbf{x})$, we know there exists $\theta(\mathbf{x}) \in \mathcal{T}$ such that

$$
0 \geq \sup _{c} \sup _{(\mathbf{a}, \mathbf{b}) \in \mathscr{C}}\left\{\Phi\left(\mathbf{F}(\mathbf{x}) \cdot \mathbf{a}+\mathbf{F}^{*}(\mathbf{x}) \cdot \mathbf{b}\right)+c \operatorname{det} \mathbf{F}(\mathbf{x})-\sum_{i=1}^{m} \theta_{i}(\mathbf{x})\left\{\Phi\left(\mathbf{U}_{i}(\mathbf{x}) \cdot \mathbf{a}+\mathbf{U}_{i}^{*}(\mathbf{x}) \cdot \mathbf{b}\right)+c \operatorname{det} \mathbf{U}_{i}(\mathbf{x})\right\}\right\} .
$$

Since $\chi^{r}(\mathbf{x}) \in\{0,1\}$ and $\sum_{r} \chi^{r}(\mathbf{x})=1$, Eq. (40) can be rewritten as

$$
0 \geq \sup _{c} \sup _{(\mathbf{a}, \mathbf{b}) \in \mathscr{C}}\left\{\Phi\left(\mathbf{F}(\mathbf{x}) \cdot \mathbf{a}+\mathbf{F}^{*}(\mathbf{x}) \cdot \mathbf{b}\right)+c \operatorname{det} \mathbf{F}(\mathbf{x})-\sum_{r=1}^{n} \sum_{i=1}^{m} \chi^{r}(\mathbf{x}) \theta_{i}(\mathbf{x})\left\{\Phi\left(\mathbf{U}_{i}^{r} \cdot \mathbf{a}+\mathbf{U}_{i}^{r * *} \cdot \mathbf{b}\right)+c \operatorname{det} \mathbf{U}_{i}^{r}\right\}\right\} .
$$

For any $r=1, \cdots, n$ and $i=1, \cdots, m$, define

$$
\theta_{i}^{r}(\mathbf{x})=\chi^{r}(\mathbf{x}) \theta_{i}(\mathbf{x})
$$

Taking volume averages in (41) yields

$$
0 \geq \sup _{c} \sup _{(\mathbf{a}, \mathbf{b}) \in \mathscr{C}}\left\{\left\langle\Phi\left(\mathbf{F} \cdot \mathbf{a}+\mathbf{F}^{*} \cdot \mathbf{b}\right)+c \operatorname{det} \mathbf{F}\right\rangle-\sum_{r=1}^{n} \sum_{i=1}^{m}\left\langle\theta_{i}^{r}\right\rangle\left\{\Phi\left(\mathbf{U}_{i}^{r} \cdot \mathbf{a}+\mathbf{U}_{i}^{r * *} \cdot \mathbf{b}\right)+c \operatorname{det} \mathbf{U}_{i}^{r}\right\}\right\}
$$

The crucial point is that the function $\mathbf{F} \mapsto \Phi\left(\mathbf{F} . \mathbf{a}+\mathbf{F}^{*} \cdot \mathbf{b}\right)+c \operatorname{det} \mathbf{F}$ is quasiconvex. Using (7) and observing from (8) that $h(\mathbf{F}) \leq \Phi\left(\mathbf{F} . \mathbf{a}+\mathbf{F}^{*} \cdot \mathbf{b}\right)+c \operatorname{det} \mathbf{F}$, we have indeed

$$
h(\overline{\mathbf{F}}) \leq\langle h(\mathbf{F})\rangle \leq\left\langle\Phi\left(\mathbf{F} . \mathbf{a}+\mathbf{F}^{*} \cdot \mathbf{b}\right)+c \operatorname{det} \mathbf{F}\right\rangle .
$$

Consider a given rotation $\overline{\mathbf{R}}$. Since $\overline{\mathbf{R}} . \mathbf{F} \in \mathscr{A}(\overline{\mathbf{R}} . \overline{\mathbf{F}})$, we obtain, in a similar way to (44),

$$
h(\overline{\mathbf{R}} \cdot \overline{\mathbf{F}}) \leq\left\langle\Phi\left(\overline{\mathbf{R}} \cdot \mathbf{F} \cdot \mathbf{a}+\overline{\mathbf{R}} \cdot \mathbf{F}^{*} \cdot \mathbf{b}\right)+c \operatorname{det}(\overline{\mathbf{R}} \cdot \mathbf{F})\right\rangle .
$$

The function $\Phi$ being frame-indifferent, the last term in (45) is equal to $\left\langle\Phi\left(\mathbf{F} \cdot \mathbf{a}+\mathbf{F}^{*} \cdot \mathbf{b}\right)+c \operatorname{det} \mathbf{F}\right\rangle$. Therefore, taking the supremum over $\overline{\mathbf{R}}$ in (45) and using the identity (8), we get

$$
\Phi\left(\overline{\mathbf{F}} \cdot \mathbf{a}+\overline{\mathbf{F}}^{*} \cdot \mathbf{b}\right)+c \operatorname{det} \overline{\mathbf{F}} \leq\left\langle\Phi\left(\mathbf{F} \cdot \mathbf{a}+\mathbf{F}^{*} \cdot \mathbf{b}\right)+c \operatorname{det} \mathbf{F}\right\rangle .
$$

That last inequality, holding for any $\overline{\mathbf{F}}$ and $\mathbf{F} \in \mathscr{A}(\overline{\mathbf{F}})$, proves that $\mathbf{F} \mapsto \Phi\left(\mathbf{F} . \mathbf{a}+\mathbf{F}^{*} . \mathbf{b}\right)$ is quasiconvex. Although it has not been stated explicitely up to this point, the quasiconvexity of $\Phi$ can be regarding as the main argument behind the bounds (10) and (27) considered in Sections 2-3. Here, in view of (43), the quasiconvexity of $\Phi$ implies that

$$
0 \geq \sup _{c} \sup _{(\mathbf{a}, \mathbf{b}) \in \mathscr{C}}\left\{\Phi\left(\overline{\mathbf{F}} \cdot \mathbf{a}+\overline{\mathbf{F}}^{*} \cdot \mathbf{b}\right)+c \operatorname{det} \overline{\mathbf{F}}-\sum_{r=1}^{n} \sum_{i=1}^{m}\left\langle\theta_{i}^{r}\right\rangle\left\{\Phi\left(\mathbf{U}_{i}^{r} \cdot \mathbf{a}+\mathbf{U}_{i}^{r ; *} \cdot \mathbf{b}\right)+c \operatorname{det} \mathbf{U}_{i}^{r}\right\}\right\} .
$$


The scalar $\left\langle\theta_{i}^{r}\right\rangle$ can be interpreted as the volume fraction of martensitic variant $i$ with orientation $r$. Note from (42) that $\left\{\left\langle\theta_{i}^{r}\right\rangle\right\}_{1 \leq i \leq m}^{1 \leq r \leq n}$ belongs to the set $\overline{\mathcal{T}}$ defined by

$$
\overline{\mathcal{T}}=\left\{\Theta \in \mathbb{R}_{m}^{n} \mid \Theta_{i}^{r} \geq 0 ; \sum_{i=1}^{m} \Theta_{i}^{r}=\left\langle\chi^{r}\right\rangle \forall r=1, \cdots, n\right\} .
$$

The developments so far show that for any $\overline{\mathbf{F}}$ in $\overline{\mathcal{K}}$, there exists $\Theta \in \overline{\mathcal{T}}$ verifying the inequality (46). This last statement can be rewritten as

$$
\overline{\mathcal{K}} \subset \overline{\mathcal{K}}_{+}
$$

where

$$
\begin{aligned}
\overline{\mathcal{K}}_{+}=\{\overline{\mathbf{F}}: & \exists \Theta \in \overline{\mathcal{T}} \text { such that } \\
& \left.0 \geq \sup _{c} \sup _{(\mathbf{a}, \mathbf{b}) \in \mathscr{C}}\left\{\Phi\left(\overline{\mathbf{F}} \cdot \mathbf{a}+\overline{\mathbf{F}}^{*} \cdot \mathbf{b}\right)+c \operatorname{det} \overline{\mathbf{F}}-\sum_{r=1}^{n} \sum_{i=1}^{m} \Theta_{i}^{r}\left\{\Phi\left(\mathbf{U}_{i}^{r} \cdot \mathbf{a}+\mathbf{U}_{i}^{r \cdot *} \cdot \mathbf{b}\right)+c \operatorname{det} \mathbf{U}_{i}^{r}\right\}\right\}\right\} .
\end{aligned}
$$

In the case where all the transformation strains have the same determinant, the set $\overline{\mathcal{K}}_{+}$can be rewritten more simply as

$$
\overline{\mathcal{K}}_{+}=\left\{\overline{\mathbf{F}}: \operatorname{det} \overline{\mathbf{F}}=\operatorname{det} \mathbf{U}_{1}^{1} ; \exists \Theta \in \overline{\mathcal{T}} \text { such that } 0 \geq \sup _{(\mathbf{a}, \mathbf{b}) \in \mathscr{C}}\left\{\Phi\left(\overline{\mathbf{F}} \cdot \mathbf{a}+\overline{\mathbf{F}}^{*} \cdot \mathbf{b}\right)-\sum_{r=1}^{n} \sum_{i=1}^{m} \Theta_{i}^{r} \Phi\left(\mathbf{U}_{i}^{r} \cdot \mathbf{a}+\mathbf{U}_{i}^{r * *} \cdot \mathbf{b}\right)\right\}\right\} .
$$

Eq. (48) means that $\overline{\mathcal{K}}_{+}$is an upper bound on the set of macroscopic energy-minimizing strains for the polycrystal. In a way similar to the bound $\overline{\mathcal{K}}_{+}^{0}$ considered in Sec. $3, \overline{\mathcal{K}}_{+}$depends on the texture through the volume fractions $\left\langle\chi^{r}\right\rangle$ of the different orientations (the later indeed appear in the definition (47) of the set $\overline{\mathcal{T}}$ ). Observe that the bound $\overline{\mathcal{K}}_{+}$is always tighter than the bound $\overline{\mathcal{K}}_{+}^{0}$ obtained from the same set $\mathscr{C}$. By (47), any $\Theta \in \overline{\mathcal{T}}$ indeed satisfies

$$
\sum_{i=1}^{m} \Theta_{i}^{r}\left(\Phi\left(\mathbf{U}_{i}^{r} \cdot \mathbf{a}+\mathbf{U}_{i}^{r * *} \cdot \mathbf{b}\right)+c \operatorname{det} \mathbf{U}_{i}^{r}\right) \leq\left\langle\chi^{r}\right\rangle \max _{1 \leq i \leq m}\left\{\Phi\left(\mathbf{U}_{i}^{r} \cdot \mathbf{a}+\mathbf{U}_{i}^{r, *} \cdot \mathbf{b}\right)+c \operatorname{det} \mathbf{U}_{i}^{r}\right\}
$$

for $r=1, \cdots, n$. It follows that $\overline{\mathcal{K}}_{+} \subset \overline{\mathcal{K}}_{+}^{0}$. We also note that, contrary to the bound $\overline{\mathcal{K}}_{+}^{0}$, the bound $\overline{\mathcal{K}}_{+}$in (49) coincides with the monocrystalline bound $\tilde{\mathcal{K}}_{+}$in the homogeneous limit. This shows that the inclusion $\overline{\mathcal{K}}_{+} \subset \overline{\mathcal{K}}_{+}^{0}$ can be strict, i.e. that the bound $\overline{\mathcal{K}}_{+}$can bring a genuine improvement.

Regarding the practical calculation of the bound $\overline{\mathcal{K}}_{+}$, observe that the inequality $0 \geq \Phi\left(\overline{\mathbf{F}} \cdot \mathbf{a}+\overline{\mathbf{F}}^{*} . \mathbf{b}\right)-$ $\sum_{r, i} \Theta_{i}^{r} \Phi\left(\mathbf{U}_{i}^{r} \cdot \mathbf{a}+\mathbf{U}_{i}^{r, *} \cdot \mathbf{b}\right)$ in (49) can be viewed as a linear constraint on $\Theta$, parametrized by $\overline{\mathbf{F}}$. Deformation gradients $\overline{\mathbf{F}}$ in $\overline{\mathcal{K}}_{+}$are characterized by the fact that those linear constraints (supplemented by the conditions $\Theta \in \overline{\mathcal{T}}$ ) define a non empty set of $\mathbb{R}_{m}^{n}$. In the language of linear programming, this amounts to detect feasibility of the linear constraints [31], which is not a direct calculation - even for a discrete $\mathscr{C}$. In the next section, we show how the problem can be conveniently solved in the case of a two-orientation / three-well polycrystal.

\section{Analytical example}

\subsection{Upper bounds}

We consider a polycrystal with two orientations, assuming without loss of generality that orientation 1 is the reference orientation. The constitutive single crystals obey a cubic to tetragonal transformation. We are interested in estimating the energy-minimizing strains at $T<T^{0}$, i.e. in the case where $\mathcal{K}^{1}=\bigcup_{i=1}^{3} \operatorname{SO}(3) \mathbf{U}_{i}^{1}$ with $\mathbf{U}_{1}^{1}, \mathbf{U}_{2}^{1}, \mathbf{U}_{3}^{1}$ given by (30). The set $\mathcal{K}^{2}$ of strains that minimize the microscopic free energy in orientation 2 can be written as $\mathcal{K}^{2}=\mathbf{R}^{2} . \mathcal{K}^{1}{ }^{t} \mathbf{R}^{2}$ where $\mathbf{R}^{2} \in \mathrm{SO}(3)$. 
Table 1 Values of $(\mathbf{a}, \mathbf{b})$ used for the cubic to tetragonal transformation

\begin{tabular}{cccc}
\hline $\mathbf{a}$ & $\mathbf{b}$ & $\Phi_{k}^{1}(\mathbf{a}, \mathbf{b})$ & $\Phi_{i}^{1}(\mathbf{a}, \mathbf{b})(i \neq k)$ \\
\hline $\mathbf{v}_{k} \otimes \mathbf{v}_{k}$ & 0 & $\eta_{2}$ & $\eta_{1}$ \\
0 & $\mathbf{v}_{k} \otimes \mathbf{v}_{k}$ & $\eta_{1}^{2}$ & $\eta_{1} \eta_{2}$ \\
$\left(\mathbf{v}_{i} \pm \mathbf{v}_{j}\right) \otimes \mathbf{v}_{k}$ & 0 & $\sqrt{2} \eta_{1}$ & $\sqrt{\eta_{1}^{2}+\eta_{2}^{2}}$ \\
0 & $\left(\mathbf{v}_{i} \pm \mathbf{v}_{j}\right) \otimes \mathbf{v}_{k}$ & $\sqrt{2} \eta_{1} \eta_{2}$ & $\eta_{1} \sqrt{\eta_{1}^{2}+\eta_{2}^{2}}$ \\
$\frac{\eta_{1}}{\eta_{1}^{2}-\eta_{2}^{2}} \mathbf{v}_{k} \otimes \mathbf{v}_{k}$ & $-\frac{\eta_{2}}{\eta_{1}\left(\eta_{1}^{2}-\eta_{2}^{2}\right)} \mathbf{v}_{k} \otimes \mathbf{v}_{k}$ & 0 & 1 \\
$\frac{\eta_{2}}{\eta_{1}^{2}-\eta_{2}^{2}} \mathbf{v}_{k} \otimes \mathbf{v}_{k}$ & $-\frac{1}{\eta_{1}^{2}-\eta_{2}^{2}} \mathbf{v}_{k} \otimes \mathbf{v}_{k}$ & 1 & 0 \\
\hline
\end{tabular}

We first illustrate how the prescription (49) can be used to derive a closed-form bound on $\overline{\mathcal{K}}$. By (49) we know that for any given $\overline{\mathbf{F}}$ in $\overline{\mathcal{K}}$, there exists $\Theta \in \overline{\mathcal{T}}$ verifying

$$
0 \geq \Phi\left(\overline{\mathbf{F}} . \mathbf{a}+\overline{\mathbf{F}}^{*} \cdot \mathbf{b}\right)-\sum_{r=1}^{2} \sum_{l=1}^{3} \Theta_{l}^{r} \Phi_{l}^{r}(\mathbf{a}, \mathbf{b})
$$

with the notation $\Phi_{l}^{r}(\mathbf{a}, \mathbf{b})=\Phi\left(\mathbf{U}_{l}^{r} \cdot \mathbf{a}+\mathbf{U}_{l}^{r, *} \cdot \mathbf{b}\right)$. Assume we can pick out $(\mathbf{a}, \mathbf{b})$ such that

$$
\Phi_{i}^{1}(\mathbf{a}, \mathbf{b})=\Phi_{j}^{1}(\mathbf{a}, \mathbf{b}) \neq \Phi_{k}^{1}(\mathbf{a}, \mathbf{b})
$$

for some permutation $(i, j, k)$ of $(1,2,3)$. Since $\sum_{l=1}^{3} \Theta_{l}^{1}=\left\langle\chi^{1}\right\rangle$, the relation (52) gives

$$
0 \geq \Phi\left(\overline{\mathbf{F}} \cdot \mathbf{a}+\overline{\mathbf{F}}^{*} \cdot \mathbf{b}\right)+\Theta_{k}^{1}\left(\Phi_{i}^{1}(\mathbf{a}, \mathbf{b})-\Phi_{k}^{1}(\mathbf{a}, \mathbf{b})\right)-\left\langle\chi^{1}\right\rangle \Phi_{i}^{1}(\mathbf{a}, \mathbf{b})-\sum_{l=1}^{3} \Theta_{l}^{2} \Phi_{l}^{2}(\mathbf{a}, \mathbf{b})
$$

Using the fact that $\Theta_{l}^{2} \geq 0$ and $\sum_{l=1}^{3} \Theta_{l}^{2}=\left\langle\chi^{2}\right\rangle$, we get

$$
0 \geq \Phi\left(\overline{\mathbf{F}} \cdot \mathbf{a}+\overline{\mathbf{F}}^{*} \cdot \mathbf{b}\right)+\Theta_{k}^{1}\left(\Phi_{i}^{1}(\mathbf{a}, \mathbf{b})-\Phi_{k}^{1}(\mathbf{a}, \mathbf{b})\right)-\left\langle\chi^{1}\right\rangle \Phi_{i}^{1}(\mathbf{a}, \mathbf{b})-\left\langle\chi^{2}\right\rangle \max _{1 \leq l \leq 3} \Phi_{l}^{2}(\mathbf{a}, \mathbf{b}) .
$$

That last inequality can be rewritten as

$$
\begin{aligned}
& \Theta_{k}^{1} \leq \frac{-\Phi\left(\overline{\mathbf{F}} \cdot \mathbf{a}+\overline{\mathbf{F}}^{*} \cdot \mathbf{b}\right)+\left\langle\chi^{1}\right\rangle \Phi_{i}^{1}(\mathbf{a}, \mathbf{b})+\left\langle\chi^{2}\right\rangle \max _{l} \Phi_{l}^{2}(\mathbf{a}, \mathbf{b})}{\Phi_{i}^{1}(\mathbf{a}, \mathbf{b})-\Phi_{k}^{1}(\mathbf{a}, \mathbf{b})} \text { if } \Phi_{i}^{1}(\mathbf{a}, \mathbf{b})-\Phi_{k}^{1}(\mathbf{a}, \mathbf{b})>0, \\
& \Theta_{k}^{1} \geq \frac{-\Phi\left(\overline{\mathbf{F}} \cdot \mathbf{a}+\overline{\mathbf{F}}^{*} \cdot \mathbf{b}\right)+\left\langle\chi^{1}\right\rangle \Phi_{i}^{1}(\mathbf{a}, \mathbf{b})+\left\langle\chi^{2}\right\rangle \max _{l} \Phi_{l}^{2}(\mathbf{a}, \mathbf{b})}{\Phi_{i}^{1}(\mathbf{a}, \mathbf{b})-\Phi_{k}^{1}(\mathbf{a}, \mathbf{b})} \text { if } \Phi_{i}^{1}(\mathbf{a}, \mathbf{b})-\Phi_{k}^{1}(\mathbf{a}, \mathbf{b})<0 .
\end{aligned}
$$

Observe that tensors $(\mathbf{a}, \mathbf{b})$ of the form (31) verify the condition (53). In a separate study of the monocrystalline cubic to tetragonal transformation [24], other tensors $(\mathbf{a}, \mathbf{b})$ verifying (53) have been found. Those tensors $(\mathbf{a}, \mathbf{b})$ are listed in Table 1, along with the corresponding values of $\Phi_{l}^{1}(\mathbf{a}, \mathbf{b})$. The tensors $(\mathbf{a}, \mathbf{b})$ in Table 1 have been found in the course of a full optimization of (10) with respect to tensors $(\mathbf{a}, \mathbf{b})$ that are diagonal in the reference cubic lattice (see [24] for more details).

Substituting the values of Table 1 in (54), we find that

$$
A_{k}^{1}(\overline{\mathbf{F}}) \leq \Theta_{k}^{1} \leq B_{k}^{1}(\overline{\mathbf{F}})
$$

where $A_{k}^{1}(\overline{\mathbf{F}})$ and $B_{k}^{1}(\overline{\mathbf{F}})$ are explicit functions whose expressions are reported in Appendix A. A similar analysis can be performed to the orientation $r=2$. This is simply achieved by replacing $\mathbf{v}_{k}$ by $\mathbf{R}^{2} \cdot \mathbf{v}_{k}$ and swapping the roles of orientations 1 and 2 in the previous developments. Such a procedure leads to

$$
A_{k}^{2}(\overline{\mathbf{F}}) \leq \Theta_{k}^{2} \leq B_{k}^{2}(\overline{\mathbf{F}})
$$



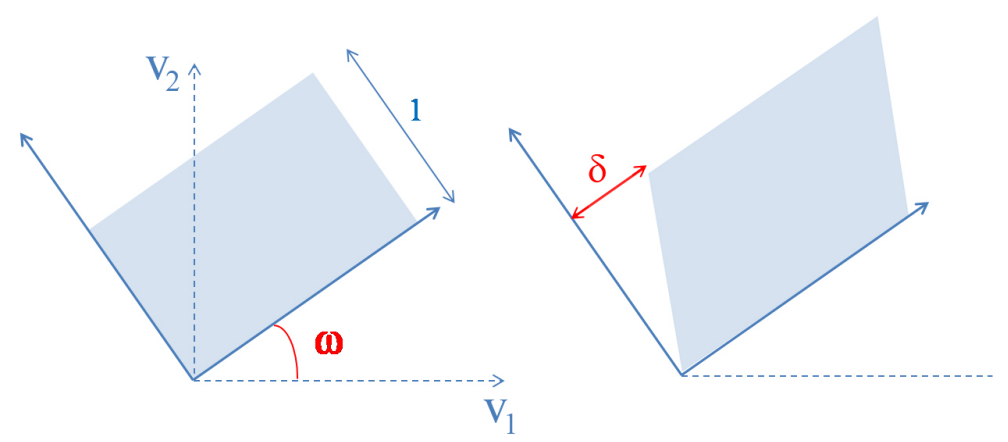

Fig. 1 Representation of the deformation $\mathbf{I}+\delta \mathbf{u}(\omega) \otimes \mathbf{v}(\omega)$ : reference (left) and deformed (right) configuration.

where the expressions of $A_{k}^{2}(\overline{\mathbf{F}})$ and $B_{k}^{2}(\overline{\mathbf{F}})$ are detailed in Appendix A. In view of (55-56), some necessary conditions for $\overline{\mathbf{F}}$ to be in $\overline{\mathcal{K}}$ are

$$
\begin{aligned}
& A_{k}^{r}(\overline{\mathbf{F}}) \leq B_{k}^{r}(\overline{\mathbf{F}}) \text { for } k=1,2,3 ; \\
& \sum_{k=1}^{3} A_{k}^{r}(\overline{\mathbf{F}}) \leq\left\langle\chi^{r}\right\rangle \leq \sum_{k=1}^{3} B_{k}^{r}(\overline{\mathbf{F}}) ;
\end{aligned}
$$

with $r=1,2$. The second inequality in (57) stems from the fact that $\sum_{k=1}^{3} \Theta_{k}^{r}=\left\langle\chi^{r}\right\rangle$. The relations (57) define the upper bound $\overline{\mathcal{K}}_{+}$corresponding to the set of tensors $(\mathbf{a}, \mathbf{b})$ listed in Table 1 (along with their rotated versions obtained by replacing $\mathbf{v}_{k}$ with $\mathbf{R}^{2} \cdot \mathbf{v}_{k}$ in Table 1).

So as to illustrate the bound obtained, consider deformation gradients $\mathbf{F}(\omega, \delta)$ of the form

$$
\mathbf{F}(\omega, \delta)=\left(\eta_{1}^{2} \eta_{2}\right)^{1 / 3}(\mathbf{I}+\delta \mathbf{u}(\omega) \otimes \mathbf{v}(\omega))
$$

where

$$
\mathbf{u}(\omega)=\cos \omega \mathbf{v}_{1}+\sin \omega \mathbf{v}_{2} \text { and } \mathbf{v}(\omega)=-\sin \omega \mathbf{v}_{1}+\cos \omega \mathbf{v}_{2}
$$

The deformation gradient $\mathbf{F}(\omega, \delta)$ is a simple shear between the directions $\mathbf{u}(\omega)$ and $\mathbf{v}(\omega)$ (Figure 1), followed by a uniform dilatation $\left(\eta_{1}^{2} \eta_{2}\right)^{1 / 3} \mathbf{I}$. The parameter $\omega$ is the angle made by the shear directions $(\mathbf{u}(\omega), \mathbf{v}(\omega))$ with the directions $\left(\mathbf{v}_{1}, \mathbf{v}_{2}\right)$ of the cubic austenitic lattice in orientation 1.

In the following, we are interested in estimating the values $(\boldsymbol{\omega}, \boldsymbol{\delta})$ for which $\mathbf{F}(\boldsymbol{\omega}, \boldsymbol{\delta})$ is energy-minimizing. Except stated otherwise, all the results presented next are obtained with the lattice parameters of $\mathrm{MnCu}$, i.e. $\eta_{1}=1.0099, \eta_{2}=0.9656$ [3]. The rotation $\mathbf{R}^{2}$ defining the orientation 2 is taken as

$$
\mathbf{R}^{2}=\left(\begin{array}{ccc}
\frac{\sqrt{2}}{2} & -\frac{\sqrt{2}}{2} & 0 \\
\frac{\sqrt{2}}{2} & \frac{\sqrt{2}}{2} & 0 \\
0 & 0 & 1
\end{array}\right) \text {. }
$$

Textures satisfying the assumptions made so far (i.e. $n=2$ with $\mathbf{R}^{1}=\mathbf{I}$ and $\mathbf{R}^{2}$ given by Eq. (59) ) are observed in some ribbons of shape memory alloys [9].

Let

$$
\Delta_{+}=\left\{(\omega, \delta): \mathbf{F}(\omega, \delta) \in \overline{\mathcal{K}}_{+}\right\}
$$

be the trace of $\overline{\mathcal{K}}_{+}$on deformation gradients of the form (58). The boundary of $\Delta_{+}$is represented in Figures 2-4 (solid lines in blue) for several values of $\left\langle\chi^{1}\right\rangle$ : the deformation gradient $\mathbf{F}(\omega, \delta)$ satisfies Eq. (57) for any $(\omega, \delta)$ within the bounded domain $\Delta_{+}$delimited by the solid lines in Figures 2-4. The solid curves in Figures $2-4$ can thus be interpreted as lower and upper bounds on the shear $\delta$ for $\mathbf{F}(\omega, \delta)$ to be energy-minimizing.

Similarly, we set

$$
\Delta_{+}^{0}=\left\{(\omega, \delta): \mathbf{F}(\omega, \delta) \in \overline{\mathcal{K}}_{+}^{0}\right\}
$$

where $\overline{\mathcal{K}}_{+}^{0}$ is calculated by applying (28) with the tensors $(\mathbf{a}, \mathbf{b})$ listed in Table 1 (along with their rotated versions obtained by replacing $\mathbf{v}_{k}$ with $\mathbf{R}^{2} \cdot \mathbf{v}_{k}$ ). The boundary of $\Delta_{+}^{0}$ is plotted in dotted lines in Figures 2-4. This allows one to appreciate the improvement brought by the consideration of (49) over (27). For instance, 


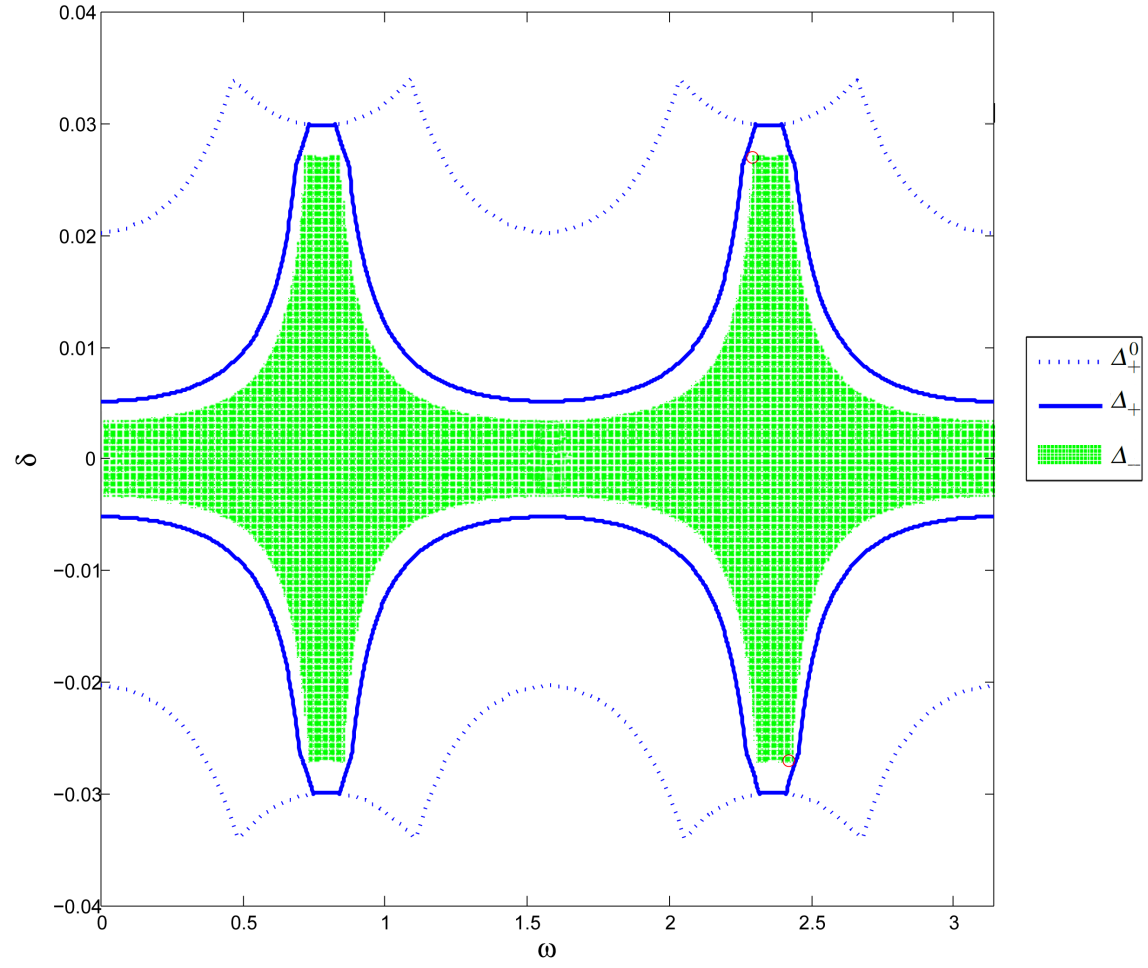

Fig. 2 Bounds on the shear $\delta$ for $\mathrm{MnCu},\left\langle\chi^{1}\right\rangle=0.9$.

in the case $\left\langle\chi^{1}\right\rangle=0.7$ (Figure 3), the relative improvement of the bound on the shear $\delta$ varies between 0 and $52 \%$ (depending on the angle $\omega$ considered), with an average of approximatively $35 \%$. In the case $\left\langle\chi^{1}\right\rangle=0.5$ (Figure 4), the relative improvement is non-negative for all $\omega$, with an average of approximatively $26 \%$.

\subsection{Lamination lower bound}

Although the fact that $\overline{\mathcal{K}}_{+}$significantly improves on $\overline{\mathcal{K}}_{+}^{0}$ is promising, it does not give any clue regarding the sharpness of the bound. The relations defining $\overline{\mathcal{K}}_{+}(49)$ are indeed necessary- but not sufficient - conditions for a deformation gradient to be energy-minimizing. The issue is to determine which deformation gradients in $\overline{\mathcal{K}}_{+}$are indeed energy-minimizing for some polycrystalline texture that is compatible with the prescribed statistics (i.e. with prescribed volume fractions of the different orientations). In order to address that question, we consider the special class of laminated textures and adapt an argument introduced in [2]: let $\mathbf{F}_{1} \in \tilde{\mathcal{K}}^{1}$ and $\mathbf{F}^{2} \in \tilde{\mathcal{K}}^{2}$ be rank-1 connected, i.e. such that

$$
\mathbf{R} . \mathbf{F}_{2}-\mathbf{F}_{1}=\mathbf{p} \otimes \mathbf{n}
$$

for some vectors $(\mathbf{p}, \mathbf{n})$ and some rotation $\mathbf{R}$. Following [2], we know that the effective deformation gradient $\left\langle\chi^{1}\right\rangle \mathbf{F}_{1}+\left(1-\left\langle\chi^{1}\right\rangle\right) \mathbf{R} . \mathbf{F}_{2}$ is energy-minimizing for an (infinitely fine) laminate texture mixing orientations 1 and 2 in proportions $\left\langle\chi^{1}\right\rangle$ and $1-\left\langle\chi^{1}\right\rangle$, respectively. The vector $\mathbf{n}$ in (60) corresponds to the normal to the interfaces in such a laminate texture. We wish to use that argument to construct values of $(\omega, \delta)$ such that $\mathbf{F}(\omega, \delta)$ is energy-minimizing for some well-chosen laminate texture. This requires to find deformation gradients $\left(\mathbf{F}_{1}, \mathbf{F}_{2}\right)$ that fulfill the two following conditions:

(1) $\mathbf{F}_{1} \in \tilde{\mathcal{K}}^{1}, \mathbf{F}_{2} \in \tilde{\mathcal{K}}^{2}$.

(2) $\mathbf{F}_{1}$ and $\mathbf{F}_{2}$ are rank-1 connected.

The condition (1) means that $\mathbf{F}_{i}$ is energy-minimizing for a single crystal with orientation $i$. The issue of finding deformation gradients that are energy-minimizing for a single crystal of tetragonal martensite has 


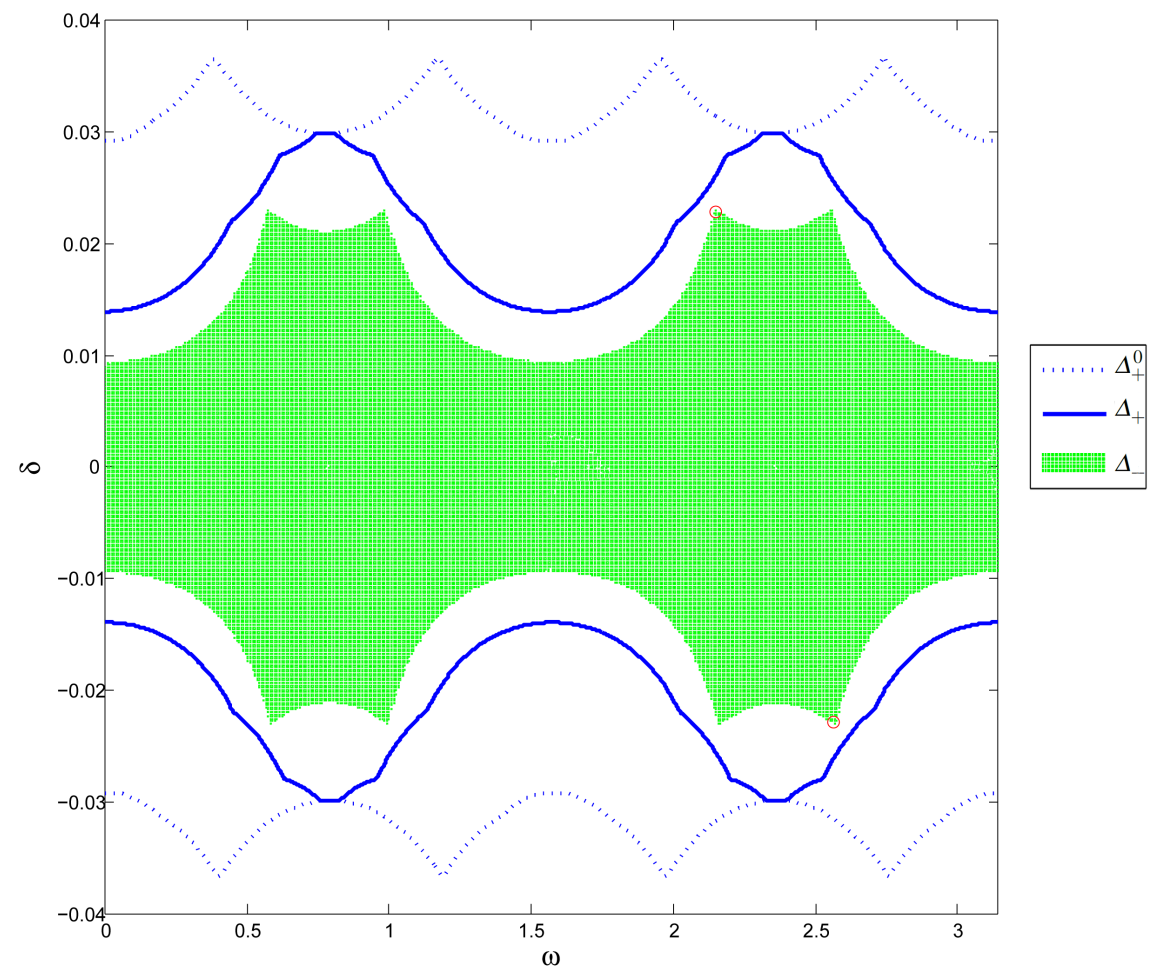

Fig. 3 Bounds on the shear $\delta$ for $\mathrm{MnCu},\left\langle\chi^{1}\right\rangle=0.7$.

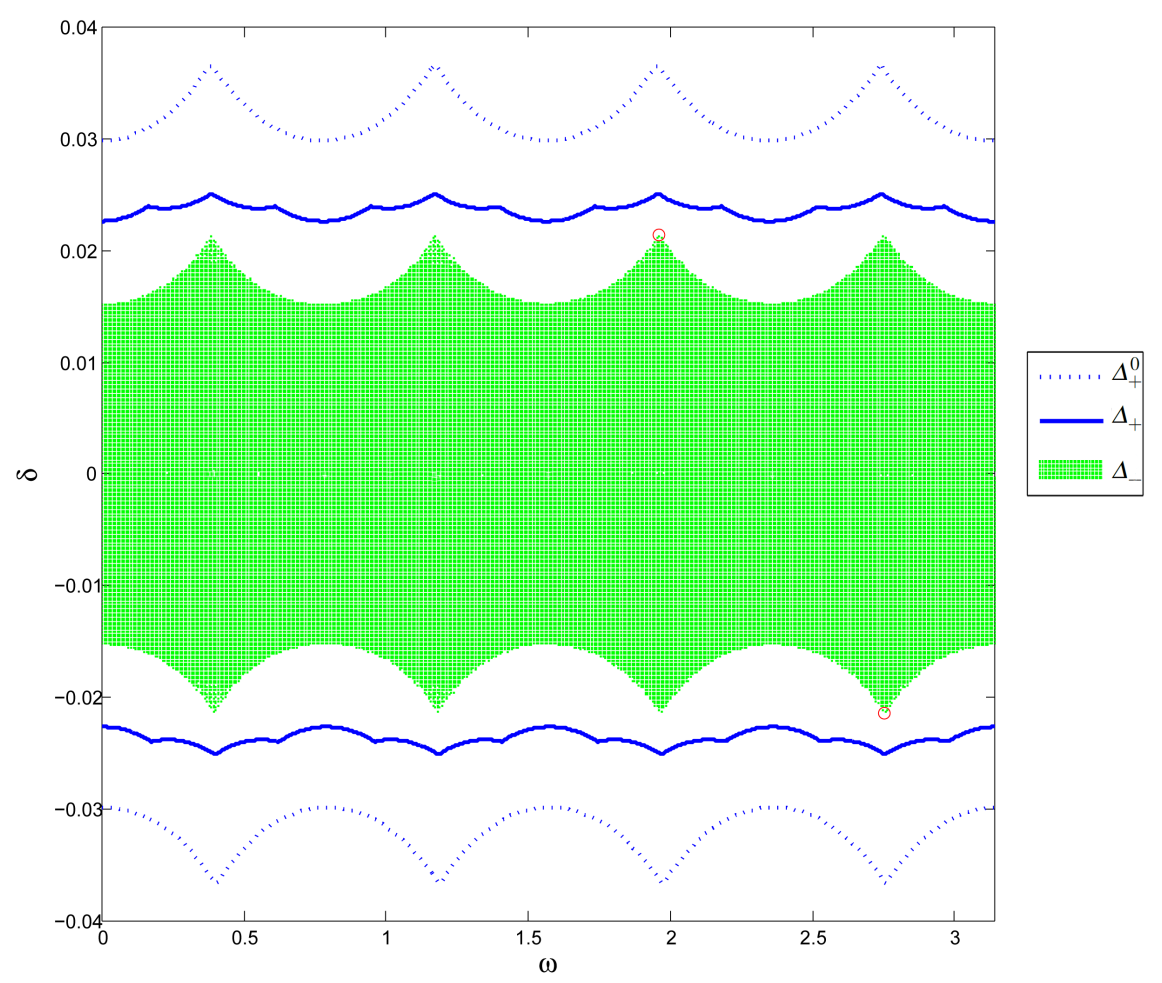

Fig. 4 Bounds on the shear $\delta$ for $\mathrm{MnCu},\left\langle\chi^{1}\right\rangle=0.5$. 
been addressed in [24]: it can be shown that $\tilde{\mathcal{K}}^{1}$ contains all the deformation gradients of the form

$$
\left(\begin{array}{ccc}
u_{11} & u_{12} & 0 \\
u_{12} & u_{22} & 0 \\
0 & 0 & \left(\eta_{1}^{2} \eta_{2}\right)^{1 / 3}
\end{array}\right)
$$

with $\left(u_{11}, u_{22}, u_{12}\right)$ verifying

$$
u_{11} u_{22}-u_{12}^{2}=\left(\eta_{1}^{2} \eta_{2}\right)^{2 / 3}, u_{11}^{2}+u_{22}^{2}+2 u_{12}^{2}+2\left|u_{12}\left(u_{11}+u_{22}\right)\right| \leq \eta_{1}^{2}+\left(\eta_{1} \eta_{2}^{2}\right)^{2 / 3} .
$$

Since $\tilde{\mathcal{K}}^{2}=\mathbf{R}^{2} . \tilde{\mathcal{K}}^{1}{ }^{t} \mathbf{R}^{2}$, it follows that $\tilde{\mathcal{K}}^{2}$ contains all the deformation gradients of the form

$$
\mathbf{R}^{2} \cdot\left(\begin{array}{ccc}
u_{11}^{\prime} & u_{12}^{\prime} & 0 \\
u_{12}^{\prime} & u_{22}^{\prime} & 0 \\
0 & 0 & \left(\eta_{1}^{2} \eta_{2}\right)^{1 / 3}
\end{array}\right) \cdot{ }^{t} \mathbf{R}^{2}
$$

where $\left(u_{11}^{\prime}, u_{22}^{\prime}, u_{12}^{\prime}\right)$ are submitted to the same restrictions as (62). As explained in [24], the deformation gradient in (61) can be realized by some fourth-rank laminated microstructure that involves the three martensitic variants.

Let now $\mathbf{F}_{1} \in \tilde{\mathcal{K}}^{1}$ and $\mathbf{F}_{2} \in \tilde{\mathcal{K}}^{2}$ be two deformation gradients of the form (61) and (63), respectively. We claim that $\mathbf{F}_{1}$ and $\mathbf{F}_{2}$ are rank-1 connected. This can be checked by applying a general procedure proposed in [1,13]: setting $\mathbf{U}_{i}=\sqrt{{ }^{t} \mathbf{F}_{i} \cdot \mathbf{F}_{i}}$ and $\mathbf{C}=\mathbf{U}_{1}^{-1} \cdot \mathbf{U}_{2}^{2} \cdot \mathbf{U}_{1}^{-1}$, given deformation gradients $\mathbf{F}_{1}$ and $\mathbf{F}_{2}$ are rank-1 connected if and only if the eigenvalues $\lambda_{1} \leq \lambda_{2} \leq \lambda_{3}$ of $\mathbf{C}$ are such that

$$
\lambda_{2}=1 \text {. }
$$

In that case, the vectors $\mathbf{p}$ and $\mathbf{n}$ solving the twinning equation (60) are given by

$$
\begin{aligned}
\mathbf{p} & =\sqrt{\frac{\lambda_{3}\left(1-\lambda_{1}\right)}{\lambda_{3}-\lambda_{1}}} \mathbf{u}_{1}+\kappa \sqrt{\frac{\lambda_{1}\left(\lambda_{3}-1\right)}{\lambda_{3}-\lambda_{1}}} \mathbf{u}_{3}, \\
\mathbf{U}_{1}^{-1} \cdot \mathbf{n} & =\left(\frac{\sqrt{\lambda_{3}}-\sqrt{\lambda_{1}}}{\sqrt{\lambda_{3}-\lambda_{1}}}\right)\left(-\sqrt{1-\lambda_{1}} \mathbf{u}_{1}+\kappa \sqrt{\lambda_{3}-1} \mathbf{u}_{3}\right)
\end{aligned}
$$

where $\kappa \in\{-1,1\}$ and $\mathbf{u}_{i}$ is a normalized eigenvector of $\mathbf{C}$ for the eigenvalue $\lambda_{i}$.

In the present case, observe from Eqs. (59)-(61)-(63) that $\operatorname{det} \mathbf{C}=1$ and $\mathbf{C} \cdot \mathbf{v}_{3}=\mathbf{v}_{3}$, so that the condition (64) is necessarily satisfied. We can thus conclude that there exists a polycrystalline texture having a volume fraction $\left\langle\chi^{1}\right\rangle$ of orientation 1 and for which the deformation gradient $\mathbf{G}$ defined by

$$
\mathbf{G}=\mathbf{F}_{1}+\left(1-\left\langle\chi^{1}\right\rangle\right) \mathbf{p} \otimes \mathbf{n}
$$

is energy-minimizing, i.e. is in the set $\overline{\mathcal{K}}$.

The final step is to observe that the deformation gradient $\mathbf{G}$ in (66) can be written as $\mathbf{G}=\mathbf{R}^{*} . \mathbf{F}\left(\omega^{*}, \delta^{*}\right)$ for some rotation $\mathbf{R}^{*}$ and some well-chosen values $\left(\omega^{*}, \delta^{*}\right)$. To that purpose, we first note that $\mathbf{v}_{3}$ being an eigenvector of the symmetric tensor $\mathbf{C}$ for the eigenvalue $\lambda_{2}=1$, the two eigenvectors $\left(\mathbf{u}_{1}, \mathbf{u}_{3}\right)$ in (65) are orthogonal to $\mathbf{v}_{3}$. It follows from the expressions (65) that the vectors $\mathbf{p}$ and $\mathbf{n}$ are also orthogonal to $\mathbf{v}_{3}$. We thus have

$$
\mathbf{G .} \mathbf{v}_{3}={ }^{\mathrm{t}} \mathbf{G} . \mathbf{v}_{3}=\left(\eta_{1}^{2} \eta_{2}\right)^{1 / 3} \mathbf{v}_{3}
$$

Using matrix representations in the basis $\left(\mathbf{v}_{1}, \mathbf{v}_{2}, \mathbf{v}_{3}\right)$, the tensor ${ }^{\mathrm{t}} \mathbf{G} . \mathbf{G} /\left(\eta_{1}^{2} \eta_{2}\right)^{2 / 3}$ can therefore be written as

$$
\left(\begin{array}{ll}
\mathbf{S} & 0 \\
0 & 1
\end{array}\right)
$$

where $\mathbf{S}$ is a symmetric positive definite matrix in $\mathbb{R}^{2 \times 2}$ with a determinant equal to 1 . Let $\left(\mu_{1}, \mu_{2}\right)$ be the eigenvalues of $\mathbf{S}$, ordered in such a way that $\mu_{1} \leq \mu_{2}$. Since $\mu_{1} \mu_{2}=\operatorname{det} \mathbf{S}=1$, we have necessarily $\mu_{1} \leq 1 \leq \mu_{2}$. Now the continuous function $\omega \mapsto \mathbf{u}(\omega)$.S.u $(\omega)$ takes values in $\left[\mu_{1}, \mu_{2}\right]$, so there exists $\omega^{*}$ verifying

$$
\mathbf{u}\left(\omega^{*}\right) . \mathbf{S} \cdot \mathbf{u}\left(\omega^{*}\right)=1 \text {. }
$$


Setting

$$
\delta^{*}=\mathbf{u}\left(\omega^{*}\right) . \mathbf{S} \cdot \mathbf{v}\left(\omega^{*}\right)
$$

the relation $\operatorname{det} \mathbf{S}=1$ implies that the matrix representation of $\mathbf{S}$ in $\left(\mathbf{u}\left(\omega^{*}\right), \mathbf{v}\left(\omega^{*}\right)\right)$ is

$$
\left(\begin{array}{cc}
1 & \delta^{*} \\
\delta^{*} & 1+\delta^{*, 2}
\end{array}\right)
$$

It follows that ${ }^{\mathrm{t}} \mathbf{G} . \mathbf{G}$ and ${ }^{\mathrm{t}} \mathbf{F}\left(\omega^{*}, \delta^{*}\right) . \mathbf{F}\left(\omega^{*}, \delta^{*}\right)$ have the same matrix representation in the basis $\left(\mathbf{u}(\omega), \mathbf{v}(\omega), \mathbf{v}_{3}\right)$, i.e. that ${ }^{\mathrm{t}} \mathbf{G} \cdot \mathbf{G}={ }^{\mathrm{t}} \mathbf{F}\left(\omega^{*}, \delta^{*}\right) \cdot \mathbf{F}\left(\omega^{*}, \delta^{*}\right)$. That last equality implies that $\mathbf{G}=\mathbf{R}^{*} \cdot \mathbf{F}\left(\omega^{*}, \delta^{*}\right)$ for some rotation $\mathbf{R}^{*}$. Since $\overline{\mathcal{K}}$ is frame-indifferent and $\mathbf{G}$ is in $\overline{\mathcal{K}}$, we can conclude that $\mathbf{F}\left(\omega^{*}, \delta^{*}\right)$ is in $\overline{\mathcal{K}}$ for the values $\left(\omega^{*}, \delta^{*}\right)$ given by (67)-(68).

As a first example of the above construction, set $s=\left(\eta_{1} / \eta_{2}\right)^{1 / 3}$ and consider the deformation gradient $\mathbf{F}_{1}^{0} \in \tilde{\mathcal{K}}^{1}$ defined by

$$
\mathbf{F}_{1}^{0}=\left(\eta_{1}^{2} \eta_{2}\right)^{1 / 3} \operatorname{diag}(s, 1 / s, 1)
$$

which satisfies (61-62). We take $\mathbf{F}_{1}=\mathbf{F}_{1}^{0}$ and $\mathbf{F}_{2}=\mathbf{R}^{2} \cdot \mathbf{F}_{1}^{0}{ }^{t} \mathbf{R}^{2}$. Setting $t=s^{4}$, the eigenvalues $\lambda_{1}$ and $\lambda_{3}$ of $\mathbf{C}$ are

$$
\lambda_{1}=\frac{1}{4 t}\left(1+2 t+t^{2}-(t-1) \sqrt{t^{2}+6 t+1}\right), \lambda_{3}=\frac{1}{4 t}\left(1+2 t+t^{2}+(t-1) \sqrt{t^{2}+6 t+1}\right)
$$

and the corresponding eigenvectors are

$$
\mathbf{u}_{1}=\mathbf{v}_{2}-\frac{\left(\sqrt{t^{2}+6 t+1}+t+1\right)}{2 t} \mathbf{v}_{1}, \mathbf{u}_{3}=\mathbf{v}_{2}+\frac{\sqrt{t^{2}+6 t+1}-t-1}{2 t} \mathbf{v}_{1} .
$$

Substituting in Eq. (65) gives two energy-minimizing strains for the polycrystal. The corresponding values of $\left(\omega^{*}, \delta^{*}\right)$ are shown as red dots in Figures 2-4. A corresponding laminate texture is shown in Figure 5(a-b) for $\left\langle\chi_{1}\right\rangle=0.7, \eta_{1}=1.5, \eta_{2}=1$. Layers of material with orientation 1 (shown in blue) are alternated with layers of material with orientation 2 (shown in red). In the reference configuration, Figure 5(a), the normal to the layers is taken as a vector $\mathbf{n}$ that is a solution of (65). In the deformed configuration, Figure 5(b), a homogeneous deformation gradient $\mathbf{F}_{1}^{0}$ (resp. $\mathbf{F}_{1}^{0}+\mathbf{p} \otimes \mathbf{n}$ ) is applied in the layers with orientation 1 (resp. 2). The macroscopic deformation gradient $\mathbf{F}\left(\omega^{*}, \delta^{*}\right)$ that is realized that way is of the form (58). In Figure 5(a), the cubic domain is chosen in a such a way that the edges of the top section are oriented along the shear directions $\left(\mathbf{u}\left(\omega^{*}\right), \mathbf{v}\left(\omega^{*}\right)\right)$.

The deformation of each monocrystalline layer in Figure 5(b) is achieved by some geometric arrangement of the martensitic variants at the microscopic level. Such a geometric arrangement is represented in Figure 5(c) for a layer with orientation 1. As detailed in Appendix, the deformation in such a layer can be achieved by a second-rank laminate involving only variants 2 and 3 (in proportion approximatively equal to 0.3338 and 0.6662 , respectively). The deformation of a layer with orientation 2 can be realized by a similar microstructure.

The procedure described so far can be repeated for any $\mathbf{F}_{1}$ and $\mathbf{F}_{2}$ of the form (61)-(63), thus generating a set of values $\left(\omega^{*}, \delta^{*}\right)$ for which $\mathbf{F}\left(\omega^{*}, \delta^{*}\right)$ is energy-minimizing. That set is denoted by $\Delta_{-}$in the following. Although difficult to carry out by hand, the calculation of $\Delta_{-}$can conveniently be performed numerically. The results are represented as green dotted domains in Figures 2-4. The corners of the domain $\Delta_{-}$correspond to all combinations of $\mathbf{F}_{1}$ in $\left\{\left(\eta_{1}^{2} \eta_{2}\right)^{1 / 3} \operatorname{diag}(s, 1 / s, 1),\left(\eta_{1}^{2} \eta_{2}\right)^{1 / 3} \operatorname{diag}(1 / s, s, 1)\right\}$ and $\mathbf{F}_{2}$ in $\mathbf{R}^{2} \cdot\left\{\left(\eta_{1}^{2} \eta_{2}\right)^{1 / 3} \operatorname{diag}(s, 1 / s, 1),\left(\eta_{1}^{2} \eta_{2}\right)^{1 / 3} \operatorname{diag}(1 / s, s, 1)\right\} .{ }^{t} \mathbf{R}^{2}$. As can be observed in Figures $2-4$, the green domain $\Delta_{-}$fills most of the domain $\Delta_{+}$, which means that most of the values of $(\omega, \delta)$ in $\Delta_{+}$can be realized by laminate textures. The gap between $\Delta_{-}$and $\Delta_{+}$could possibly be reduced by considering more complex polycrystalline textures. 


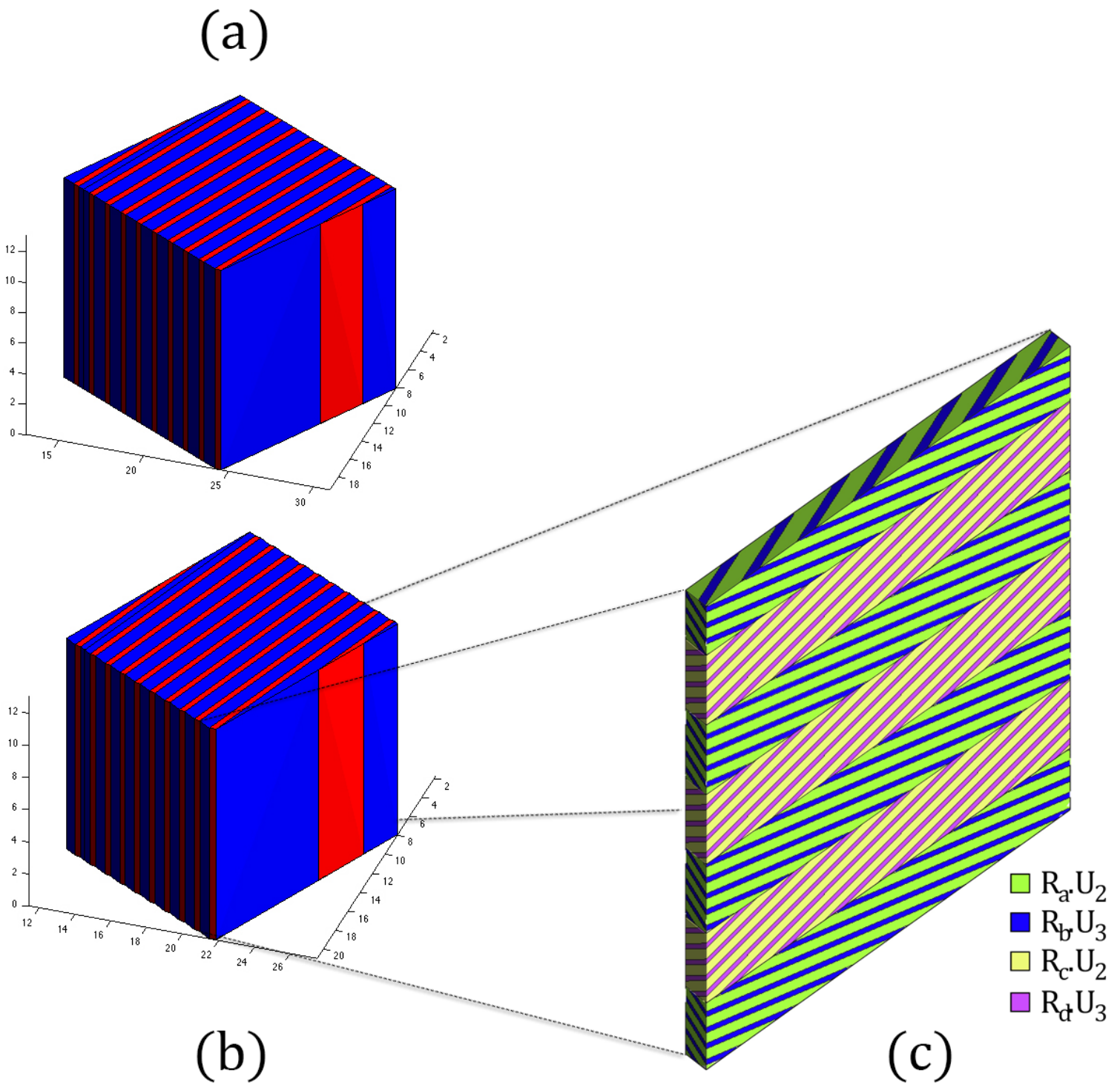

Fig. 5 Example of energy-minimizing strain in a laminated texture, $\left\langle\chi^{1}\right\rangle=0.7$ : (a) reference configuration; (b) deformed configuration; (c) details of the martensitic microstructure in a monocrystalline layer.

\subsection{Bounds on the volume fractions}

The functions $\left(A_{i}^{r}, B_{i}^{r}\right)$ that define the bound $\overline{\mathcal{K}}_{+}$in $(57)$ are of special interest as they give some information on the martensitic microstructures realizing any given energy-minimizing deformation gradient $\overline{\mathbf{F}}$. From (55), $A_{i}^{r}(\overline{\mathbf{F}})$ and $B_{i}^{r}(\overline{\mathbf{F}})$ are indeed lower and upper bounds on the volume fractions of martensitic variant $i$ in orientation $r$.

As a first example, calculating $A_{i}^{r}(\overline{\mathbf{F}})$ and $B_{i}^{r}(\overline{\mathbf{F}})$ for the deformation gradient $\overline{\mathbf{F}}$ corresponding to Figure 5 gives the restrictions

$$
\begin{aligned}
& 0 \leq \Theta_{1}^{1} \leq 0.0844, \quad 0 \leq \Theta_{1}^{2} \leq 0.1879, \\
& 0.3686 \leq \Theta_{2}^{1} \leq 0.5723,0.0329 \leq \Theta_{2}^{2} \leq 0.3 \text {, } \\
& 0.1838 \leq \Theta_{3}^{1} \leq 0.3433,0.0782 \leq \Theta_{3}^{2} \leq 0.3 \text {. }
\end{aligned}
$$


The polycrystalline texture and the martensitic microstructures displayed in Figure 5 correspond to $\Theta_{1}^{1}=0$, $\Theta_{2}^{1} \simeq 0.4662, \Theta_{3}^{1} \simeq 0.2337, \Theta_{1}^{2}=0, \Theta_{2}^{2} \simeq 0.2, \Theta_{3}^{2} \simeq 0.1$. Such values are compatible with the bounds (70), as expected.

In Figure 6 , the bounds $\left(A_{i}^{1}, B_{i}^{1}\right)$ are displayed as functions of the shear $\delta$, for several values of $\omega$. The volume fraction $\left\langle\chi^{1}\right\rangle$ is set to 0.7 . It is important to note that, because of the condition $\sum_{i} \Theta_{i}^{1}=\left\langle\chi^{1}\right\rangle$, the volume fractions $\Theta_{i}^{1}$ cannot reach any arbitrary value that is compatible with the bounds $\left(A_{i}^{1}, B_{i}^{1}\right)$. For instance in the case $\delta=0$, the upper bounds $B_{i}^{1}$ on $\Theta_{i}^{1}$ are all equal to $1 / 3$ but the volume fractions $\Theta_{i}^{1}$ cannot be simultaneously equal to the $1 / 3$ (as otherwise the constraint $\sum_{i} \Theta_{i}^{1}=\left\langle\chi^{1}\right\rangle=0.7$ would not be satisfied).

There is no general trend concerning the variations of $\left(A_{i}^{1}, B_{i}^{1}\right)$ with $\delta$. For instance, in the examples displayed in Figure 6 , the function $B_{1}^{1}$ can either be decreasing, increasing or non monotonic, depending on the value of $\omega$ considered. Some intuitive insight in the behavior of the bounds $\left(A_{i}^{1}, B_{i}^{1}\right)$ can be obtained in the infinitesimal strain approximation, using the idea that the martensitic variants that are most likely to develop are those which are the most favorably oriented with respect to the applied strain, i.e. those that maximize the quantity

$$
z_{i}(\omega, \delta)=\operatorname{tr}\left(\varepsilon_{i}^{1} \cdot \varepsilon(\omega, \delta)\right)
$$

In that last equation, $\varepsilon(\omega, \delta)=\left(\mathbf{F}(\omega, \delta)+{ }^{\mathrm{t}} \mathbf{F}(\omega, \delta)\right) / 2-\mathbf{I}$ is the infinitesimal strain associated to $\mathbf{F}(\omega, \delta)$ and $\varepsilon_{i}^{1}=\left(\mathbf{U}_{i}^{1}+{ }^{t} \mathbf{U}_{i}^{1}\right) / 2-\mathbf{I}$ is the infinitesimal transformation strain for variant $i$. For the case at hand, we find

$$
z_{1}(\omega, \delta)=\frac{\delta}{2}\left(\eta_{1}-\eta_{2}\right) \sin \omega, z_{2}(\omega, \delta)=-\frac{\delta}{2}\left(\eta_{1}-\eta_{2}\right) \sin \omega, z_{3}(\omega, \delta)=0
$$

The cases (a),(b) and (c) in Figure 6 correspond to $\sin \omega>0$. Therefore, for positive values of $\delta$, variant 1 is favorably oriented and variant 2 is unfavorably oriented. Accordingly, when $\delta$ grows from 0 , the bounds $\left(A_{1}^{1}, B_{1}^{1}\right)$ initially increase while the bounds $\left(A_{2}^{1}, B_{2}^{1}\right)$ decrease. This is in line with the intuition that more of variant 1 and less of variant 2 is expected to develop as $\delta$ increases from 0 . Since $z_{3}(\omega, \delta)=0$, variant 3 is neither favorably nor unfavorably oriented. Accordingly, the corresponding bounds $\left(A_{3}^{1}, B_{3}^{1}\right)$ remains stationary (for $\delta$ small enough). The case (d) in Figure 6 is slightly different as it corresponds to $\sin \omega=0$. In such case we have $z_{i}(\omega, \delta)=0$ for all $i$, which explains that all the bounds $\left(A_{i}^{1}, B_{i}^{1}\right)$ remains stationary. All of this reasoning is limited to the infinitesimal strain approximation, i.e. to small values for $\delta$. As $\delta$ becomes large, rotation effects become significant and the qualitative arguments explained above do no longer apply. In particular, in the case (a), we can observe that $B_{1}^{1}$ decreases for large values of $\delta$.

The transformation $\mathbf{F}(\omega, \delta)$ ceases to be energy-minimizing when one of the constraints $A_{i}^{1}<B_{i}^{1}$ or $\sum_{i} A_{i}^{1} \leq\left\langle\chi^{1}\right\rangle<\sum_{i} B_{i}^{1}$ is violated. The corresponding value of $\delta$ is displayed as a vertical dashed line in Figure 6. For instance, the constraint $A_{3}^{1}<B_{3}^{1}$ (resp. $A_{2}^{1}<B_{2}^{1}$ ) is the limiting one in the case $\omega=0.2$ (resp. $\omega=0.75$ ), see Figure 6 (a) and (b). For $\omega=1$, Figure 6(c), the limiting constraint is $\sum_{i} A_{i}^{1}<\left\langle\chi^{1}\right\rangle$. All the remarks made so far on the bounds $\left(A_{i}^{1}, B_{i}^{1}\right)$ for orientation 1 can be transposed to orientation 2 . In particular, there are some values of $\omega$ for which the limiting constraints are associated with $\left(A_{i}^{2}, B_{i}^{2}\right)$.

\section{Concluding remarks}

In this paper, some rigorous upper bounds on the energy-minimizing strains of martensitic polycrystals have been obtained in the geometrically nonlinear setting. The main results are the bounds $\overline{\mathcal{K}}_{+}^{0}$ and $\overline{\mathcal{K}}_{+}$(defined in (27) and (49) respectively) that depend on the texture through the volume fractions of the different orientations. Those bounds are expressed in terms of a given family $\mathscr{C}$ of tensors $(\mathbf{a}, \mathbf{b})$, which acts as a free parameter in (27)-(49): each choice of $\mathscr{C}$ generates corresponding bounds $\overline{\mathcal{K}}_{+}^{0}$ and $\overline{\mathcal{K}}_{+}$. For a given (say discrete) $\mathscr{C}$, the bound $\overline{\mathcal{K}}_{+}$is tighter than $\overline{\mathcal{K}}_{+}^{0}$ but more difficult to calculate: whereas checking if a given deformation gradient $\overline{\mathbf{F}}$ is in $\overline{\mathcal{K}}_{+}^{0}$ is a direct calculation, checking if $\overline{\mathbf{F}} \in \overline{\mathcal{K}}_{+}$amounts to detecting feasibility of a linear programming problem in $\mathbb{R}_{m}^{n}$. In this paper, the bounds $\overline{\mathcal{K}}_{+}^{0}$ and $\overline{\mathcal{K}}_{+}$have been used to study a 2-orientation/3-variant polycrystal, in which case the calculations could be performed in closed form (for a well chosen $\mathscr{C}$ ). For more complex textures, it is clear that numerical calculations of the bounds will be necessary at some point, which requires adequate algorithms. In that regard, it can be noted that interior-point methods offer some efficient algorithms for detecting feasibility in large-scale linear programming problems. Interestingly, such 
(a)

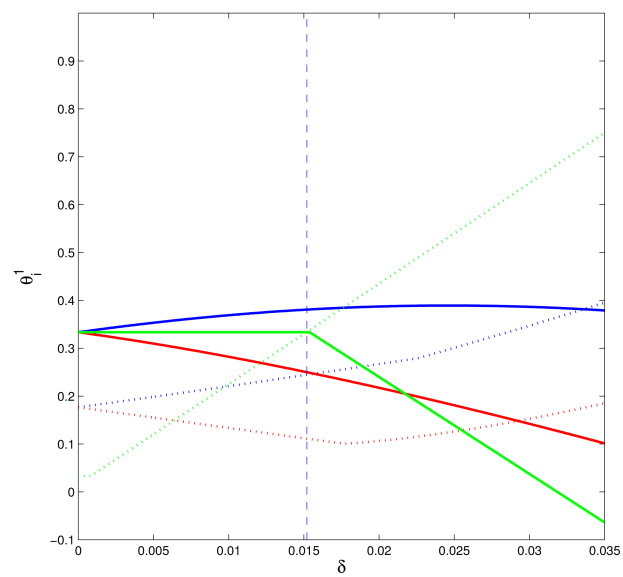

(c)

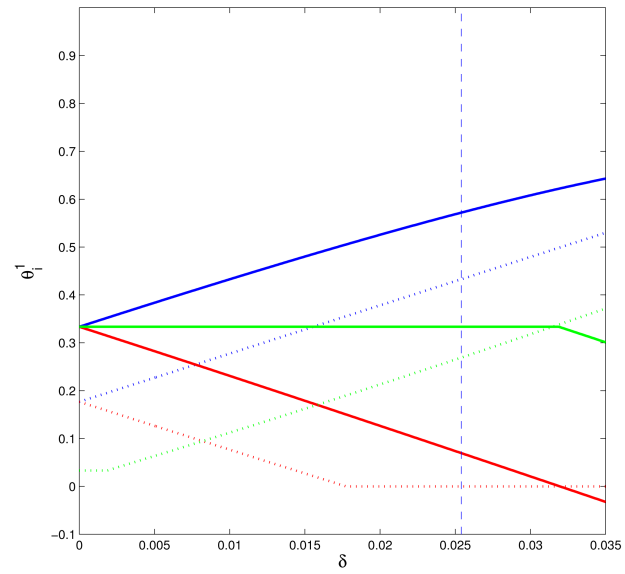

(b)

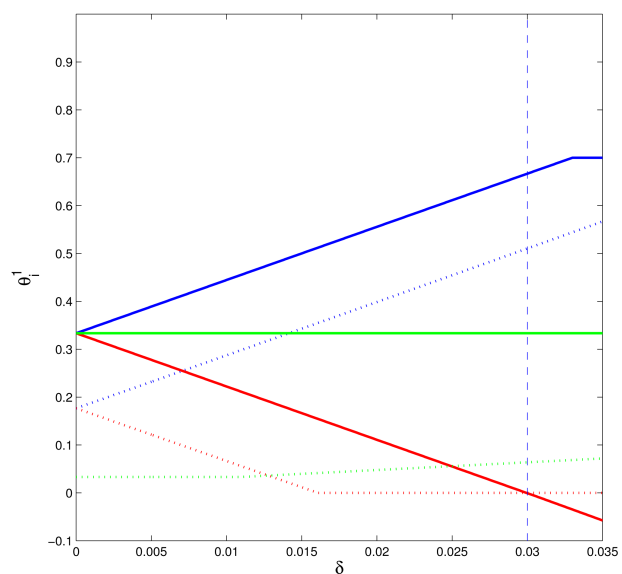

(d)

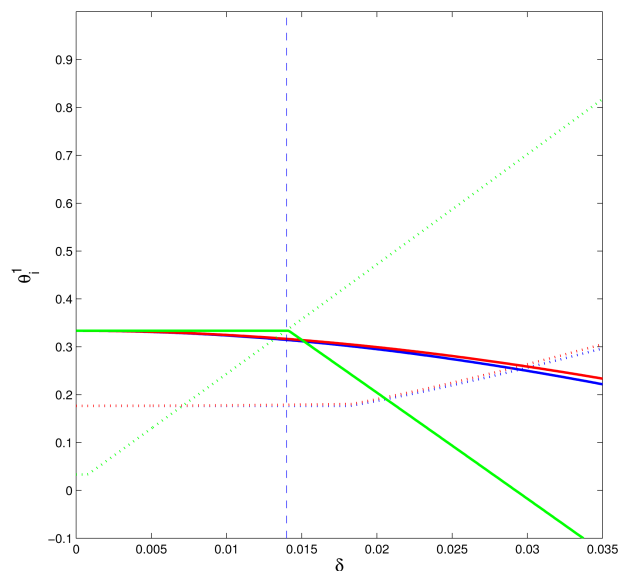

Fig. 6 Lower bound $A_{i}^{1}$ and upper bound $B_{i}^{1}$ on the volume fractions $\theta_{i}^{1}$ for $\mathrm{MnCu},\left\langle\chi^{1}\right\rangle=0.7: \omega=0.2$ (a), $\omega=0.75(\mathrm{~b}), \omega=1$ (c), $\omega=\pi / 2(\mathrm{~d})$.

algorithms, as the self-dual algorithm of Ye [32], has been used in other problems related to shape-memory alloys [26] and could possibly be useful for calculating the bound $\overline{\mathcal{K}}_{+}$in the case of a complex polycrystalline texture. A more theoretical line of investigation consists in deriving upper bounds taking more information on the texture (such as 2-point statistics) into account. 
A Bounds on the volume fractions for a two-orientation polycrystal of tetragonal martensite

Substituting the values of Table 1 in (54) and recalling that $0 \leq \Theta_{k}^{1} \leq\left\langle\chi^{1}\right\rangle$, we find that $A_{k}^{1}(\overline{\mathbf{F}}) \leq \Theta_{k}^{1} \leq B_{k}^{1}(\overline{\mathbf{F}})$ where

$$
\begin{aligned}
& A_{k}^{1}(\overline{\mathbf{F}})=\max _{\varepsilon= \pm 1}\{ \frac{\left\|\overline{\mathbf{F}}^{*} \cdot \mathbf{v}_{k}\right\|-\left\langle\chi^{1}\right\rangle \eta_{1} \eta_{2}-\left\langle\chi^{2}\right\rangle \max _{l}\left\|\mathbf{U}_{l}^{2, *} \cdot \mathbf{v}_{k}\right\|}{\eta_{1}\left(\eta_{1}-\eta_{2}\right)}, \\
& \frac{\left\|\overline{\mathbf{F}} \cdot\left(\mathbf{v}_{i}+\varepsilon \mathbf{v}_{j}\right)\right\|-\sqrt{\eta_{1}^{2}+\eta_{2}^{2}}\left\langle\chi^{1}\right\rangle-\left\langle\chi^{2}\right\rangle \max _{l}\left\|\mathbf{U}_{l}^{2} \cdot\left(\mathbf{v}_{i}+\varepsilon \mathbf{v}_{j}\right)\right\|}{\sqrt{2} \eta_{1}-\sqrt{\eta_{1}^{2}+\eta_{2}^{2}}}, \\
&\left.\frac{1}{\eta_{1}^{2}-\eta_{2}^{2}}\left(\left\|\eta_{2} \overline{\mathbf{F}} \cdot \mathbf{v}_{k}-\overline{\mathbf{F}}^{*} \cdot \mathbf{v}_{k}\right\|-\left\langle\chi^{2}\right\rangle \max _{l}\left\|\eta_{2} \mathbf{U}_{l}^{2} \cdot \mathbf{v}_{k}-\mathbf{U}_{l}^{2, *} \cdot \mathbf{v}_{k}\right\|\right), 0\right\} ; \\
& B_{k}^{1}(\overline{\mathbf{F}})=\min _{\varepsilon= \pm 1}\left\{\frac{\eta_{1}\left\langle\chi^{1}\right\rangle-\left\|\overline{\mathbf{F}} \cdot \mathbf{v}_{k}\right\|+\left\langle\chi^{2}\right\rangle \max _{l}\left\|\mathbf{U}_{l}^{2} \cdot \mathbf{v}_{k}\right\|}{\eta_{1}-\eta_{2}},\right. \\
& \frac{\eta_{1} \sqrt{\eta_{1}^{2}+\eta_{2}^{2}}\left\langle\chi^{1}\right\rangle-\left\|\overline{\mathbf{F}}^{*} \cdot\left(\mathbf{v}_{i}+\varepsilon \mathbf{v}_{j}\right)\right\|+\left\langle\chi^{2}\right\rangle \max _{l}\left\|\mathbf{U}_{l}^{2, *} \cdot\left(\mathbf{v}_{i}+\varepsilon \mathbf{v}_{j}\right)\right\|}{\eta_{1}\left(\sqrt{\eta_{1}^{2}+\eta_{2}^{2}}-\sqrt{2} \eta_{2}\right)}, \\
&\left.\left\langle\chi^{1}\right\rangle-\frac{1}{\eta_{1}\left(\eta_{1}^{2}-\eta_{2}^{2}\right)}\left(\left\|\eta_{1}^{2} \overline{\mathbf{F}} \cdot \mathbf{v}_{k}-\eta_{2} \overline{\mathbf{F}}^{*} \cdot \mathbf{v}_{k}\right\|-\left\langle\chi^{2}\right\rangle \max _{l}\left\|\eta_{1}^{2} \mathbf{U}_{l}^{2} \cdot \mathbf{v}_{k}-\eta_{2} \mathbf{U}_{l}^{2, *} \cdot \mathbf{v}_{k}\right\|\right),\left\langle\chi^{1}\right\rangle\right\} .
\end{aligned}
$$

In those expressions, the indexes $(i, j)$ are such that $(i, j, k)$ is a permutation of $(1,2,3)$. Replacing $\mathbf{v}_{k}$ by $\mathbf{R}^{2} \cdot \mathbf{v}_{k}$ and swapping the roles of orientations 1 and 2, we obtain in a similar fashion that $A_{k}^{2}(\overline{\mathbf{F}}) \leq \theta_{k}^{2} \leq B_{k}^{2}(\overline{\mathbf{F}})$ with

$$
\begin{aligned}
& A_{k}^{2}(\overline{\mathbf{F}})=\max _{\varepsilon= \pm 1}\left\{\frac{\left\|\overline{\mathbf{F}}^{*} \cdot \mathbf{R}^{2} \cdot \mathbf{v}_{k}\right\|-\left\langle\chi^{2}\right\rangle \eta_{1} \eta_{2}-\left\langle\chi^{1}\right\rangle \max _{l}\left\|\mathbf{U}_{l}^{1, *} \cdot \mathbf{R}^{2} \cdot \mathbf{v}_{k}\right\|}{\eta_{1}\left(\eta_{1}-\eta_{2}\right)}\right. \\
& \frac{\left\|\overline{\mathbf{F}} \cdot \mathbf{R}^{2} \cdot\left(\mathbf{v}_{i}+\varepsilon \mathbf{v}_{j}\right)\right\|-\sqrt{\eta_{1}^{2}+\eta_{2}^{2}}\left\langle\chi^{2}\right\rangle-\left\langle\chi^{1}\right\rangle \max _{l}\left\|\mathbf{U}_{l}^{1} \cdot\left(\mathbf{R}^{2} \cdot \mathbf{v}_{i}+\varepsilon \mathbf{R}^{2} \cdot \mathbf{v}_{j}\right)\right\|}{\sqrt{2} \eta_{1}-\sqrt{\eta_{1}^{2}+\eta_{2}^{2}}}, \\
& \left.\frac{1}{\eta_{1}^{2}-\eta_{2}^{2}}\left(\left\|\eta_{2} \overline{\mathbf{F}} \cdot \mathbf{R}^{2} \cdot \mathbf{v}_{k}-\overline{\mathbf{F}}^{*} \cdot \mathbf{R}^{2} \cdot \mathbf{v}_{k}\right\|-\left\langle\chi^{1}\right\rangle \max _{l}\left\|\eta_{2} \mathbf{U}_{l}^{1} \cdot \mathbf{R}^{2} \cdot \mathbf{v}_{k}-\mathbf{U}_{l}^{1, *} \cdot \mathbf{R}^{2} \cdot \mathbf{v}_{k}\right\|\right), 0\right\} ; \\
& B_{k}^{2}(\overline{\mathbf{F}})=\min _{\varepsilon= \pm 1}\left\{\frac{\eta_{1}\left\langle\chi^{2}\right\rangle-\left\|\overline{\mathbf{F}} \cdot \mathbf{R}^{2} \cdot \mathbf{v}_{k}\right\|+\left\langle\chi^{1}\right\rangle \max _{l}\left\|\mathbf{U}_{l}^{1} \cdot \mathbf{R}^{2} \cdot \mathbf{v}_{k}\right\|}{\eta_{1}-\eta_{2}},\right. \\
& \frac{\eta_{1} \sqrt{\eta_{1}^{2}+\eta_{2}^{2}}\left\langle\chi^{2}\right\rangle-\left\|\overline{\mathbf{F}}^{*} \cdot\left(\mathbf{R}^{2} \cdot \mathbf{v}_{i}+\varepsilon \mathbf{R}^{2} \cdot \mathbf{v}_{j}\right)\right\|+\left\langle\chi^{1}\right\rangle \max _{l}\left\|\mathbf{U}_{l}^{1, *} \cdot\left(\mathbf{R}^{2} \cdot \mathbf{v}_{i}+\varepsilon \mathbf{R}^{2} \cdot \mathbf{v}_{j}\right)\right\|}{\eta_{1}\left(\sqrt{\eta_{1}^{2}+\eta_{2}^{2}}-\sqrt{2} \eta_{2}\right)}, \\
& \left.\left\langle\chi^{2}\right\rangle-\frac{1}{\eta_{1}\left(\eta_{1}^{2}-\eta_{2}^{2}\right)}\left(\left\|\eta_{1}^{2} \overline{\mathbf{F}} \cdot \mathbf{R}^{2} \cdot \mathbf{v}_{k}-\eta_{2} \overline{\mathbf{F}}^{*} \cdot \mathbf{R}^{2} \cdot \mathbf{v}_{k}\right\|-\left\langle\chi^{1}\right\rangle \max _{l}\left\|\eta_{1}^{2} \mathbf{U}_{l}^{1} \cdot \mathbf{R}^{2} \cdot \mathbf{v}_{k}-\eta_{2} \mathbf{U}_{l}^{1, *} \cdot \mathbf{R}^{2} \cdot \mathbf{v}_{k}\right\|\right),\left\langle\chi^{2}\right\rangle\right\} .
\end{aligned}
$$

Although somewhat lengthy, the obtained expressions are fully explicit and easy to calculate.

\section{B Construction of a microstructure realizing the deformation gradient $\mathbf{F}_{1}^{0}$ in Eq. (69)}

In this Appendix, we determine a martensitic microstructure realizing the deformation gradient $\mathbf{F}_{1}^{0}$ in (69) for a reference single crystal with transformation strains given by (30). To that purpose, we first observe that $\mathbf{F}_{1}^{0}$ is in the quasiconvex hull of $\operatorname{SO}(3) \mathbf{U}_{2} \cup$ $\mathrm{SO}(3) \mathbf{U}_{3}$ (which is denoted by $\tilde{\mathcal{K}}_{23}$ from now on). Following [2], symmetric positive definite tensors $\mathbf{U}$ in $\tilde{\mathcal{K}}_{23}$ are indeed characterized by matrix representations (in the basis $\left(\mathbf{v}_{1}, \mathbf{v}_{2}, \mathbf{v}_{3}\right)$ of the cubic austenitic lattice) of the form

$$
\left(\begin{array}{ccc}
\eta_{1} & 0 & 0 \\
0 & u_{22} & u_{23} \\
0 & u_{23} & u_{33}
\end{array}\right)
$$

with $u_{22} u_{33}-u_{23}^{2}=\eta_{1} \eta_{2}$ and

$$
u_{22}^{2}+u_{33}^{2}+2 u_{23}^{2}+2\left|u_{23}\left(u_{22}+u_{33}\right)\right| \leq \eta_{1}^{2}+\eta_{2}^{2} .
$$

The fact that $\mathbf{F}_{1}^{0}$ is in $\tilde{\mathcal{K}}_{23}$ means that $\mathbf{F}_{1}^{0}$ can be realized by a microstructure involving variants 2 and 3 only. Also observe from the characterization given above that symmetric positive definite tensors $\mathbf{U}$ in $\tilde{\mathcal{K}}_{23}$ can be parametrized by their components $\left(u_{23}, u_{22}\right)$. In Figure 7 is represented the set of values $\left(u_{23}, u_{22}\right)$ corresponding to symmetric positive definite tensors $\mathbf{U}$ in $\tilde{\mathcal{K}}_{23}$. 


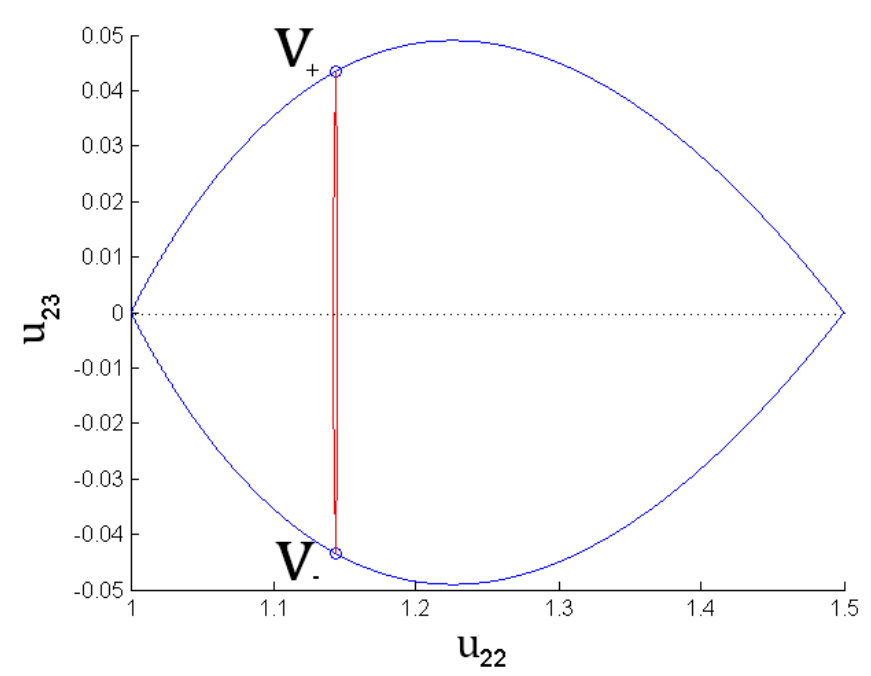

Fig. 7 Representation of $\tilde{\mathcal{K}}_{23}$.

Now consider a deformation gradient $\mathbf{V}_{+}$on the boundary of the domain in Figure 7, and let $\mathbf{V}_{-}$be its symmetric with respect to the $u_{23}=0$ axis. We can write

$$
\mathbf{V}_{+}=\left(\begin{array}{ccc}
\eta_{1} & 0 & 0 \\
0 & v_{22} & v_{23} \\
0 & v_{23} & v_{33}
\end{array}\right), \mathbf{v}_{-}=\left(\begin{array}{ccc}
\eta_{1} & 0 & 0 \\
0 & v_{22} & -v_{23} \\
0 & -v_{23} & v_{33}
\end{array}\right)
$$

and we assume that $v_{23} \geq 0$. Using the fact that $\mathbf{V}_{+}$satisfies (71) as an equality, we can relate $\left(v_{22}, v_{33}\right)$ to $v_{23}$ by the expressions

$$
v_{22}=\frac{1}{2}\left(S+\varepsilon \sqrt{S^{2}-4 P}\right), v_{33}=\frac{1}{2}\left(S-\varepsilon \sqrt{S^{2}-4 P}\right)
$$

with $S=-v_{23}+\sqrt{v_{23}^{2}+\left(\eta_{1}+\eta_{2}\right)^{2}}, P=\eta_{1} \eta_{2}+v_{23}^{2}$ and $\varepsilon= \pm 1$.

Following [2], deformation gradients on the boundary of the domain in Figure 7 can be realized by a simple laminate of variants 2 and 3, i.e. there exists $c_{ \pm}$in $[0,1]$ and $\mathbf{R}_{ \pm} \in \mathrm{SO}(3)$ such that

$$
\mathbf{V}_{ \pm}=\mathbf{R}_{ \pm} \cdot\left(\mathbf{U}_{2}+\left(1-c_{ \pm}\right) \mathbf{b} \otimes \mathbf{n}\right)
$$

where $(\mathbf{b}, \mathbf{n})$ are solutions of the twinning equation $\left(\mathbf{U}_{2}+\mathbf{b} \otimes \mathbf{n}\right) \cdot \mathbf{U}_{3}^{-1} \in \operatorname{SO}(3)$. There are actually two sets of vectors $(\mathbf{b}, \mathbf{n})$ solving the twinning equation. We denote them by $\left(\mathbf{b}_{+}, \mathbf{n}_{+}\right)$and $\left(\mathbf{b}_{-}, \mathbf{n}_{-}\right)$. For $\left(\eta_{1}, \eta_{2}\right)=(1.5,1)$, we have $\mathbf{n}_{+}=x\left(\mathbf{v}_{2}-\mathbf{v}_{3}\right)$ and $\mathbf{n}_{-}=-x\left(\mathbf{v}_{2}+\mathbf{v}_{3}\right)$ with $x \simeq 0.6934$. It turns out that $\mathbf{V}_{+}$satisfies (73) with $(\mathbf{b}, \mathbf{n})=\left(\mathbf{b}_{+}, \mathbf{n}_{+}\right)$, while $\mathbf{V}_{-}$satisfies (73) with $(\mathbf{b}, \mathbf{n})=\left(\mathbf{b}_{-}, \mathbf{n}_{-}\right)$.

Eq. (73) expresses the fact that $\mathbf{V}_{ \pm}$is realized by a laminate with a direction of lamination equal to $\mathbf{n}$, mixing variants 2 and 3 in proportion $c_{ \pm}$and $1-c_{ \pm}$respectively. As $\mathbf{V}_{+}$and $\mathbf{V}_{-}$are symmetric with respect to the $u_{23}=0$ axis in Figure 7 , it can be proved that $c_{+}=c_{-}$. The exact value of $c_{+}$depends on the deformation gradient $\mathbf{V}_{+}$considered.

The same argument as used in Section 6.2 shows that $\mathbf{V}_{+}$and $\mathbf{V}_{-}$are rank-1 connected. Therefore $\tilde{\mathcal{K}}_{23}$ contains the deformation gradient

$$
\mathbf{H}(c)=\mathbf{V}_{+}+(1-c) \mathbf{b}^{\prime} \otimes \mathbf{n}^{\prime}
$$

where $\left(\mathbf{b}^{\prime}, \mathbf{n}^{\prime}\right)$ solve the twinning equation $\left(\mathbf{V}_{+}+\mathbf{b}^{\prime} \otimes \mathbf{n}^{\prime}\right) . \mathbf{V}_{-}^{-1} \in \mathrm{SO}(3)$. The parameter $c$ can take any value in $[0,1]$. Let $s$ be the operator that maps any given $\mathbf{F}$ in $G L_{+}(3)$ to its symmetric definite positive part $\mathbf{U}$ in the polar decomposition $\mathbf{F}=\mathbf{R}$.U. When $c$ varies from 0 to 1, the tensor $s(\mathbf{H}(c))$ remains in $\tilde{\mathcal{K}}_{23}$ and varies between $\mathbf{V}_{+}$and $\mathbf{V}_{-}$, as represented in Figure 7 (red lines). Since the twinning equation $\left(\mathbf{V}_{+}+\mathbf{b}^{\prime} \otimes \mathbf{n}^{\prime}\right) . \mathbf{V}_{-}^{-1} \in \mathrm{SO}(3)$ generally admits two sets of solutions $\left(\mathbf{b}^{\prime}, \mathbf{n}^{\prime}\right)$, there are two branches $c \mapsto s(\mathbf{H}(c))$ to be considered, as can be seen in Figure 7. Independently on the branch considered, it can be verified that

$$
\mathbf{v}_{2} . s(\mathbf{H}(1 / 2)) \cdot \mathbf{v}_{3}=0,
$$

i.e. the curve $v \mapsto s(\mathbf{H}(c))$ in Figure 7 crosses the $u_{23}=0$ axis for $c=1 / 2$. It follows that $s(\mathbf{H}(1 / 2))$ as a matrix representation of the form $\operatorname{diag}\left(\eta_{1}, y, \eta_{1} \eta_{2} / y\right)$ with $y=\mathbf{v}_{2} . s(\mathbf{H}(1 / 2)) \cdot \mathbf{v}_{2}$. Hence $s(\mathbf{H}(1 / 2))$ is equal to $\mathbf{F}_{1}^{0}$ if

$$
\mathbf{v}_{2} \cdot s(\mathbf{H}(1 / 2)) \cdot \mathbf{v}_{2}=\left(\eta_{1} \eta_{2}^{2}\right)^{1 / 3} .
$$

Recall that $\mathbf{H}(1 / 2)$ depends on $\mathbf{V}_{+}$, the latter being parametrized by $v_{23}$. The function $v_{23} \mapsto \mathbf{v}_{2} . s(\mathbf{H}(1 / 2))$. $\mathbf{v}_{2}$ is represented in Figure 8 for one of the branches $c \mapsto \mathbf{H}(c)$, with $\varepsilon=1$ in (72). As can be observed on Figure 8, the condition (74) is satisfied for 


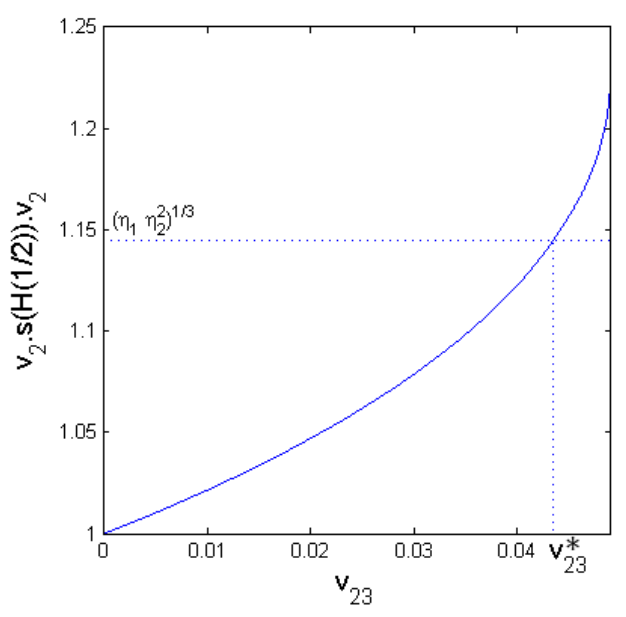

Fig. 8 Determination of the microstructural parameter $v_{23}$.

some value $v_{23}^{*}$ of the parameter $v_{23}$ (numerical calculations give $v_{23}^{*} \simeq 0.04352$ ). The corresponding values of $c_{+}, \mathbf{V}_{+}, \mathbf{b}^{\prime}, \mathbf{n}^{\prime}$ are denoted by $c_{+}^{*}, \mathbf{V}_{+}^{*}, \mathbf{b}^{\prime *}, \mathbf{n}^{\prime *}$, respectively. In particular, we note that $c_{+}^{*} \simeq 0.3338$ and $\mathbf{n}^{\prime *} \simeq-0.1868 \mathbf{v}_{3}$.

The conclusion is that the deformation gradient $\mathbf{F}_{1}^{0}$ can be realized by a laminate of normal $\mathbf{n}^{* *}$, mixing the deformation gradients $\mathbf{V}_{+}^{*}$ and $\mathbf{V}_{+}^{*}+(1 / 2) \mathbf{b}^{\prime *} \otimes \mathbf{n}^{\prime *}$ in equal proportion. Since $\mathbf{V}_{+}^{*}$ and $\mathbf{V}_{+}^{*}+(1 / 2) \mathbf{b}^{\prime *} \otimes \mathbf{n}^{\prime *}$ are themselves realized by firstrank laminates of variants 2 and 3 (with the same volume fraction $c_{+}^{*}$ ), the deformation gradient $\mathbf{F}_{1}^{0}$ is realized by a second-rank laminate of variant 2 (in volume fraction $c_{+}^{*}$ ) and variant 3 . Such a second-rank laminate microstructure is displayed in Figure $5(c)$.

\section{References}

1. Ball, J., James, R.: Fine phase mixtures as minimizers of energy. Arch. Rational Mech. Anal. 100, 13-52 (1987)

2. Ball, J., James, R.: Proposed experimental tests of a theory of fine microstructure and the two-well problem. Phil. Trans. Roy. Soc. London A. 338, 338-450 (1992)

3. Basinski, Z., Christian, J.: The cubic tetragonal transformation in manganese copper alloys. Journal of the Institute of Metals 80(12), 659-666 (1952)

4. Bhattacharya, K.: Microstructure of Martensite. Oxford University Press (2003)

5. Bhattacharya, K., Dolzmann, G.: Relaxation on some multi-well problems. Proc. Royal Soc. Edin A 131, 279-320 (2001)

6. Bhattacharya, K., Kohn, R.: Energy minimization and the recoverable strains in polycrystalline shape memory alloys. Arch Rational Mech. Anal. 139, 99-180 (1997)

7. Chenchiah, I.V., Schlömerkemper, A.: Non-laminate microstructures in monoclinic-i martensite. Arch. Rational Mech. Anal. 207(1), 39-74 (2013)

8. Dacorogna, B.: Direct methods in the calculus of variations, second edition. Springer (2008)

9. Eucken, S., Hirsch, J.: The effect of textures on shape memory behaviour. In: Materials Science Forum, vol. 56, pp. 487-492. Trans Tech Publ (1991)

10. Govindjee, S., Hackl, K., Heinen, R.: An upper bound to the free energy of mixing by twin-compatible lamination for n-variant martensitic phase transformations. Continuum Mech. Thermodyn. 18, 443-453 (2007)

11. Govindjee, S., Mielke, A., Hall, G.J.: The free energy of mixing for $n$-variant martensitic phase transformations using quasi-convex analysis-variant martensitic phase transformations using quasi-convex analysis. J.Mech.Phys.Solids 51(4), I-XXVI (2003)

12. Hackl, K., Heinen, R.: An upper bound to the free energy of $n$-variant polycrystalline shape memory alloys. J.Mech.Phys.Solids 56, 2832-2843 (2008)

13. Khachaturyan, A.: Theory of Structural Transformation in solids. John Wiley \& Sons, New York (1983)

14. Kinderlehrer, D., Pedregal, P.: Characterizations of young measures generated by gradients. Arch. Rational Mech. Anal. 115, 329-365 (1991)

15. Kohn, R.: Relaxation of a double-well energy. Continuum Mech. Thermodyn. 3, 193-236 (1991)

16. Milton G.W.: The theory of composites. Cambridge University Press (2002)

17. Milton, G.W., Serkov, S.K.: Bounding the current in nonlinear conducting composites. J. Mech. Phys. Solids 48, 1295-1324 (2000)

18. Misra, A., Singh, V.: Thermomechanics-based nonlinear rate-dependent coupled damage-plasticity granular micromechanics model. DOI:10.1007/s00161-014-0360-y, Continuum Mech. Thermodyn., 1-31 (2014)

19. Müller, S.: Variational models for microstructure and phase transitions. In: Calculus of variations and geometric evolution problems. Springer (1999)

20. Otsuka, K., Wayman, C.: Shape memory materials. Cambridge University Press (1998) 
21. Peigney M.: A pattern-based method for bounding the effective response of a nonlinear composite. J. Mech. Phys. Solids 53, 923-948 (2005)

22. Peigney, M.: Recoverable strains in composite shape-memory alloys. J. Mech. Phys. Solids 56, 360-375 (2008)

23. Peigney, M.: A non-convex lower bound on the effective free energy of polycrystalline shape memory alloys. J. Mech. Phys. Solids 57, 970-986 (2009)

24. Peigney, M.: On the energy-minimizing strains in martensitic microstructures-part 1: Geometrically nonlinear theory. J.Mech.Phys.Solids 61, 1489-1510 (2013)

25. Peigney, M.: On the energy-minimizing strains in martensitic microstructures-part 2: Geometrically linear theory. J.Mech.Phys.Solids 61, 1511-1530 (2013)

26. Peigney, M., Seguin, J., Hervé-Luanco, E.: Numerical simulation of shape memory alloys structures using interior-point methods. Int. J. Sol. Struct. 48(20), 2791-2799 (2011)

27. Pipkin, A.C.: Elastic materials with two preferred states. Q. J. Mechanics Appl. Math. 44(1), 1-15 (1991)

28. Shu, Y., Bhattacharya, K.: The influence of texture on the shape-memory effects in polycrystals. Acta Mater. 15, 5457-5473 (1998)

29. Smyshlyaev, V., Willis, J.R.: On the relaxation of a three-well energy. Proc. R. Soc. Lond. A 455, 779-814 (1998)

30. Talbot, D.R.S., Willis, J.R.: Bounds for the effective constitutive relation of a nonlinear composite. Proc. R. Soc. Lond. A 460, 2705-2723 (2004)

31. Ye, Y.: Interior point algorithms: theory and analysis. Wiley Interscience (1997)

32. Ye, Y.: On homogeneous and self-dual algorithm for linear complementarity problems. Mathematical Programming 76, 211-222 (1997)

33. Zhao, L., Willemse, P.F., Mulder, J.H., Beyer, J., Wei,W.:Texture development and transformation strain of a cold-rolled Ti50-Ni45-Cu5 alloy Scripta Mater. 39, 1317-1323 (1998) 